\title{
Metabolic Potential, Ecology and Presence of Associated Bacteria Is Reflected in Genomic Diversity of Mucoromycotina
}

\section{OPEN ACCESS}

Edited by:

David W. Ussery,

University of Arkansas for Medical

Sciences, United States

Reviewed by:

Seraphim Papanikolaou

Agricultural University of Athens,

Greece

Somayeh Dolatabadi,

Westerdijk Fungal Biodiversity

Institute, Netherlands

${ }^{*}$ Correspondence:

Anna Muszewska

musze@ibb.waw.pl;

ania.muszewska@gmail.com

Specialty section:

This article was submitted to

Evolutionary and Genomic

Microbiology,

a section of the journal

Frontiers in Microbiology

Received: 02 December 2020

Accepted: 25 January 2021

Published: 15 February 2021

Citation:

Muszewska A, Okrasińska A

Steczkiewicz K, Drgas O,

Orłowska M, Perlińska-Lenart U, Aleksandrzak-Piekarczyk T, Szatraj K,

Zielenkiewicz U, Piłsyk S, Malc E,

Mieczkowski P, Kruszewska JS, Bernat $P$ and Pawłowska J (2021)

Metabolic Potential, Ecology and Presence of Associated Bacteria

Is Reflected in Genomic Diversity

of Mucoromycotina.

Front. Microbiol. 12:636986

doi: 10.3389/fmicb.2021.636986
Anna Muszewska ${ }^{1 *}$, Alicja Okrasińska ${ }^{2}$, Kamil Steczkiewicz', Olga Drgas' ${ }^{1}$, Małgorzata Orłowska ${ }^{1}$, Urszula Perlińska-Lenart', Tamara Aleksandrzak-Piekarczyk', Katarzyna Szatraj', Urszula Zielenkiewicz', Sebastian Piłsyk' ${ }^{1}$, Ewa Malc ${ }^{3}$, Piotr Mieczkowski ${ }^{3}$, Joanna S. Kruszewska ${ }^{1}$, Przemysław Bernat ${ }^{4}$ and Julia Pawłowska ${ }^{2}$

${ }^{1}$ Institute of Biochemistry and Biophysics, Polish Academy of Sciences, Warsaw, Poland, ${ }^{2}$ Institute of Evolutionary Biology, Faculty of Biology, Biological and Chemical Research Centre, University of Warsaw, Warsaw, Poland, ${ }^{3}$ High Throughput Sequencing Facility of UNC, Chapel Hill, NC, United States, ${ }^{4}$ Department of Industrial Microbiology and Biotechnology, Faculty of Biology and Environmental Protection, University of Łódź, Łódź, Poland

Mucoromycotina are often considered mainly in pathogenic context but their biology remains understudied. We describe the genomes of six Mucoromycotina fungi representing distant saprotrophic lineages within the subphylum (i.e., Umbelopsidales and Mucorales). We selected two Umbelopsis isolates from soil (i.e., U. isabellina, $U$. vinacea), two soil-derived Mucor isolates (i.e., M. circinatus, M. plumbeus), and two Mucorales representatives with extended proteolytic activity (i.e., Thamnidium elegans and Mucor saturninus). We complement computational genome annotation with experimental characteristics of their digestive capabilities, cell wall carbohydrate composition, and extensive total lipid profiles. These traits inferred from genome composition, e.g., in terms of identified encoded enzymes, are in accordance with experimental results. Finally, we link the presence of associated bacteria with observed characteristics. Thamnidium elegans genome harbors an additional, complete genome of an associated bacterium classified to Paenibacillus sp. This fungus displays multiple altered traits compared to the remaining isolates, regardless of their evolutionary distance. For instance, it has expanded carbon assimilation capabilities, e.g., efficiently degrades carboxylic acids, and has a higher diacylglycerol:triacylglycerol ratio and skewed phospholipid composition which suggests a more rigid cellular membrane. The bacterium can complement the host enzymatic capabilities, alter the fungal metabolism, cell membrane composition but does not change the composition of the cell wall of the fungus. Comparison of early-diverging Umbelopsidales with evolutionary younger Mucorales points at several subtle differences particularly in their carbon source preferences and encoded carbohydrate repertoire. Nevertheless, all tested Mucoromycotina share features including the ability to produce 18:3 gamma-linoleic acid, use TAG as the storage lipid and have fucose as a cell wall component.

Keywords: Mucorales, Umbelopsidales, Paenibacillus, comparative genomics, carbon source usage, lipid profile, cell wall carbohydrates 


\section{INTRODUCTION}

Mucoromycotina subphylum comprises three orders: Umbelopsidales, Endogonales and Mucorales (Spatafora et al., 2016). While Umbelopsidales and Mucorales group mostly saprotrophic fungi living in the soil, on dung and litter, Endogonales are known to establish symbiotic interactions with plants (Desirò et al., 2017). The ancestors of extant Mucoromycotina were among the first colonizers of land. These early-branching fungi possess all of the traits commonly acknowledged as distinctive characteristics of the fungal kingdom such as apical growth, presence of ergosterol in the membranes and a cell wall made of chitin and betaglucan (Richards et al., 2017). Yet, their cell wall differs from ascomycetes and basidiomycetes by the presence of fucose and high amounts of $\mathrm{N}$-acetylglucosamine and glucuronic acid (Bartnicki-Garcia, 1968; Mélida et al., 2015) which is typical for other Opisthokonta rather than fungi. Mucoromycotina representatives form a fast-growing mycelium of haploid hyphae. The sexual phase includes the fusion of two gametangia and formation of a resting spore called zygospore (Spatafora et al., 2016).

Apart from decomposing organic matter as saprotrophs, some Mucoromycotina are capable of forming mutualistic and mycorrhiza-like associations with Haplomitriopsida liverworts (Bidartondo et al., 2011; Field et al., 2015). Others are parasites of plants and animals (PartidaMartinez and Hertweck, 2005; Ibrahim and Spellberg, 2014). The ecology of Mucoromycotina is poorly studied, which hinders understanding of their role in the ecosystem (Richardson and Rautemaa-Richardson, 2019).

The best-studied order of Mucoromycotina is Mucorales. Its representatives are perceived mostly as a source of food spoilage, rotting crops and decomposing litter (Morin-Sardin et al., 2017). Due to their efficient plant matter degrading potential, Mucorales are used in various food production processes like the fermentation of soya beans known in the traditional Asian and African cuisine as tempeh (Nout et al., 2010) or cheese making (Hermet et al., 2012). Others are employed as bio producers of polyunsaturated fatty acids (e.g., M. circinelloides), $\beta$-carotene (e.g., Blakeslea trispora, M. circinelloides, and Phycomyces blakesleeanus) and diverse hydrolases (Roukas et al., 2015; Voigt et al., 2016; Rivaldi et al., 2017). Certain Mucorales (e.g., M. indicus) are also able to produce ethanol (Satari and Karimi, 2018) while others (e.g., Rhizomucor miehei) are commercially used for lipase production (Morin-Sardin et al., 2017; Meghwanshi and Vashishtha, 2018). Mucorales lipid composition includes gamma-linolenic fatty acid 18:3 (da Silva et al., 1998) which is found mostly in plants (Mukherjee and Kiewitt, 1987), algae (Nichols and Wood, 1968) and not in Dikarya which produce the n-3 isomer of the C18 trienoic fatty acid alpha-linolenic acid.

Some Mucorales are involved in life-threatening, opportunistic infections with mortality rates reaching up to 40-80\% (Cornely et al., 2019). There are general traits which predispose microorganisms to become opportunistic pathogens, e.g., thermotolerance and ability to evade immune cells. Many non-pathogenic fungi are adapted to higher temperatures due to living in decomposing organic matter, often warmed up due to rotting processes (Maheshwari et al., 2000; Neher et al., 2013), which allows them to survive inside the animal warm-blooded body.

Recently, endohyphal bacteria have been described in a few different fungal lineages of Mucoromycota (i.e., Mucoromycotina and 2 other closely related subphyla: Glomeromycotina and Mortierellomycotina). Mycoplasma-related endobacteria (MRE) were detected in Endogonales (Chang et al., 2019), Glomeromycotina (Desirò et al., 2014), and Mortierellomycotina (Desirò et al., 2018). Burkholderia-related endobacteria (BRE) were identified in Mortierellomycotina, Mucorales and Gigasporales representatives (Bonfante and Venice, 2020). Paraburkholderia-related endobacteria (PRE) were discovered in Mucorales, Umbelopsidales, and Mortierellomycotina representatives (Okrasińska et al., 2021). Lately, information about two other endosymbionts of Rhizopus microsporus has been published: Ralstonia pickettii as an facilitator of fungal invasion during infection (Itabangi et al., 2019) and Stenotrophomonas sp. whose role has not yet been established (Virgianti and Natalia, 2020). The activity of endohyphal and fungi-associated bacteria involves changes in lipid metabolism (Lumini et al., 2007; Lastovetsky et al., 2016; Uehling et al., 2017) changes in morphology of the fungus (Lumini et al., 2007; Salvioli et al., 2016; Uehling et al., 2017) control the hosts' sexual (Mondo et al., 2017), and asexual reproduction (Partida-Martinez et al., 2007).

Here we describe the genomes of six Mucoromycotina species representing separated saprotrophic lineages within the subphylum (i.e., Umbelopsidales and Mucorales). Despite numerous studies on Mucoromycotina in a pathogenic context their basic biology remains understudied. We selected two Umbelopsis isolates from soil: $U$. isabellina and $U$. vinacea, two soil-derived Mucor isolates: M. circinatus and M. plumbeus, and two Mucorales representatives: Thamnidium elegans and M. saturninus with proteolytic capabilities enabling them to colonize dung and animal substrate (Hanagasaki and Asato, 2018). Two strains were obtained from the CBSKNAW culture collection and the remaining four were selected from the Mucoromycotina collection of University of Warsaw Herbarium. We complement genome analysis with a phenotypic description of their digestive potential, their cell wall carbohydrate composition, and total lipid profiles. Finally, we link the presence of endohyphal bacteria with observed characteristics.

\section{RESULTS}

\section{Genome Assembly and Annotation}

Obtained assemblies showed diverse levels of fragmentation depending on genome size and abundance of repeats. Both Umbelopsis genomes assembled into fewer than 200 scaffolds whereas Mucor assemblies were significantly more fragmented (Table 1).

Genome completeness was verified using single copy fungal orthologous genes searched by BUSCO (Simão et al., 2015). 
TABLE 1 | Summary of genome sequence characteristics.

\begin{tabular}{|c|c|c|c|c|c|c|c|}
\hline & $\begin{array}{l}\text { Umbelopsis } \\
\text { isabellina }\end{array}$ & $\begin{array}{c}\text { Umbelopsis } \\
\text { vinacea }\end{array}$ & $\begin{array}{c}\text { Mucor } \\
\text { circinatus }\end{array}$ & $\begin{array}{c}\text { Mucor } \\
\text { plumbeus }\end{array}$ & $\begin{array}{c}\text { Mucor } \\
\text { saturninus }\end{array}$ & $\begin{array}{c}\text { Thamnidium } \\
\text { elegans }\end{array}$ & Paenibacillus sp. \\
\hline Genome size [Mb] & 22 & 23.2 & 55.7 & 49.5 & 40.3 & 31 & 6.9 \\
\hline Genes & 9030 & 9241 & 14148 & 11690 & 13020 & 10765 & 5954 \\
\hline tRNAs & 129 & 134 & 194 & 274 & 262 & 191 & 119 \\
\hline TE number & 37 & 59 & 1681 & 869 & 1166 & 541 & - \\
\hline$\%$ GC & 42 & 43.09 & 31.89 & 31.1 & 36.59 & 33.15 & 44.61 \\
\hline$\%$ repeats & 7.85 & 7.81 & 28.24 & 43.3 & 32.52 & 18 & - \\
\hline Contigs & 82 & 175 & 16245 & 8313 & 7254 & 3243 & 285 \\
\hline Coverage (x) & 60 & 68 & 26 & 28 & 31 & 61 & 8 \\
\hline N50 & 1335078 & 1328092 & 125937 & 49105 & 159134 & 100281 & 129168 \\
\hline L50 & 7 & 7 & 116 & 301 & 68 & 89 & 16 \\
\hline BUSCOS \% & 98.3 & 97.6 & 96.9 & 97.2 & 98.7 & 94.5 & 85.4 \\
\hline
\end{tabular}

Only a few BUSCOSs were duplicated from 27 in U. isabellina up to 57 in $M$. saturninus suggests that sequenced genomes were not duplicated. The number of predicted genes, repeats and transposable elements (TEs) content correlates positively with genome size.

\section{Repetitive Elements}

The largest group of TEs found in the genomes of Mucorales, with more than 200 copies per genome are class II elements belonging to Tc1/Mariner superfamily (Supplementary Table 1) but other DNA-repeats were also ubiquitous. EnSpm, PIF/Harbinger, hAT-Ac MuLE, Merlin, PiggyBac were present from one up to a hundred copies in all sequenced Mucorales. EnSpm, PIF/Harbinger, hAT-Ac and MuLE are ubiquitous in fungi in general, whereas Merlin elements are characteristic for basal fungal lineages and were apparently lost in Dikarya (Muszewska et al., 2017b). Unlike Mucorales, Umbelopsidales genomes had just a few copies of Tc1/Mariner, hAT-Ac, and a single Ginger element. Class I retrotransposon landscape is dominated by LTR retrotransposons from Ty3/Gypsy and LINE/L1 elements which were found in all genomes except for $U$. vinacea which lacks the L1s. Surprisingly the omnipresent Ty1/Copia elements were identified only in the genome of M. plumbeus (two copies). Also, all sequenced species had LTR/DIRS elements, characteristic for their tyrosinase integrase and are absent from Dikarya (Muszewska et al., 2013). Mucor genomes harbor also numerous Helitrons, known for their rolling circle replication mechanism. The presence of Helitrons has been observed in other Mucor species previously (Lebreton et al., 2020). Noteworthy, Helitrons often hijack neighboring genes and are efficient vectors of HGT in other fungi (Castanera et al., 2014). Overall, Umbelopsis genomes contained ten-fold less transposable elements per genome compared to Mucorales, which is expected taking into account the differences in genome size.

\section{RNAi and Other Defense Mechanisms}

Fungal genomes are usually protected via diverse mechanisms that include fungal repeat-induced point mutation (RIP), methylation induced premeiotically (MIP), meiotic silencing of unpaired DNA (MSUD) and quelling. It has been described that Mucormycotina possesses RNAi pathway components (Cervantes et al., 2013) which are also important for fungus biology (Nicolás et al., 2015; Calo et al., 2017). We scanned the sequenced genomes with HMM profiles of RNAi core enzymes, namely Dicer, Argonaute and RdRP, and demonstrate that all are present in all six fungal genomes, with duplication of RdRP in all Mucorales (from three to five copies per genome) and a single copy in both Umbelopsis isolates (Supplementary Table 2). $U$. vinacea, M. circinatus, M. plumbeus harbored duplicated Argonaute proteins.

Self-non-self and the fungal immune system based on Nucleotide Oligomerization Domain (NOD)-like receptors (NLRs) could be identified neither in the six genomes described in this work nor in 14 predicted Mucormycotina proteomes available at NCBI. The typical NLR central domains NACHT or NB-ARC seem to be absent from those genomes.

\section{Detection of Paenibacillus Bacteria in Thamnidium Genome}

Initial Thamnidium assembly contained genome fragments characterized by two clearly distinct GC content ratios and for that reason was additionally analyzed as a metagenome. It is composed of two easily distinguishable fractions, one belonging to a presumed fungal host and the latter to an associated bacterium representing Paenibacillus, Firmicutes. Fungal and bacterial genomes were re-assembled separately. Remaining five Mucoromycotina assemblies did not contain significant amounts of sequences with sequence similarity to non-Mucoromycotina taxa.

\section{Paenibacillus Features}

The bacterial genome is moderately complete (BUSCO score 85.7\%) and encodes 5815 genes. Its closest relative, Paenibacillus sp. 7523-1, despite DDH similarity above 70\% threshold for two out of three DDH calculation formulas implemented in TYGS $(67.2 ; 88.3 ; 72.9)$, differs in GC content by $2.35 \%$ which supports the separation of the newly sequenced strain as a new species (Supplementary Figure 1 and Supplementary Table 11). A phylogenetic tree inferred from 16S RNA of related isolates 
shows its proximity to $P$. illinoensis isolates despite differences in GC content (Figure 1). GC content of the identified Paenibacillus genome could be altered by misplaced fungal reads. Despite discarding all reads mapping on eukaryote genomes when assembling the Paenibacillus genome it is still possible that some fungal fragments, not similar to other eukaryotic sequences, could not be filtered out.

The bacterial genome has only one complete antimicrobial locus, a rifamycin-inactivating phosphotransferase $\mathrm{RphD}$, identified by scanning using ABRicate. We found a partial beta-lactam resistance pathway lacking the central betalactamase (four copies of beta-N-acetylhexosaminidase, BlaI family transcriptional regulator, oligopeptide transport system ATP-binding protein from oppA to oppF, penicillin-binding protein $1 \mathrm{~A}$ and $2 \mathrm{~A})$. Additionally, the Peanibacillus genome encodes a single M56 peptidase and 15 proteins bearing an S12 domain. Family M56 includes BlaR1 which is the antirepressor in beta-lactamase operon (Zhang and Chambers, 2004). Family S12 groups carboxypeptidases B vital for cell wall synthesis and remodeling, as well as beta-lactamases (Tang, 2018). Taken together, all these traces point at a potential beta-lactam resistance of Paenibacillus. Glycopeptide antibiotic resistance may also be present in some form since partial vancomycin resistance operon was also identified, once more only regulation and accessory components are preserved. The possible activity of these proteins and their relevance for resistance is not known.
The Paenibacillus genome harbors vitamin biosynthesis pathways, e.g., cobalamin (B12), riboflavin (B2), menaquinone (K2), and thiamine (B1). It also has all the necessary genes for molybdenum cofactor synthesis and manipulation. Paenibacillus has many quorum sensing genes and those coding for flagellar and mobility proteins. Its metabolic potential is described below in parallel with its fungal host and remaining fungal genomes.

The Paenibacillus genome encodes 3 copies of Peptidase_U32 (PF01136), a collagenase that may facilitate meat degradation by Thamnidium elegans which is one of the few fungi known for this property (Dashdorj et al., 2016). This protein is found mostly in Firmicutes and is absent from eukaryotic genomes. The U32 collagenases are considered as virulence factors in animalinfecting bacteria (Navais et al., 2014). The U32 collagenase (PrtC) together with urease subunit alpha (UreB) are parts of the Helicobacter pylori arsenal used in epithelial cell invasion (Kavermann et al., 2003). Paenibacillus sp. genome harbors all three urease subunits (alpha, beta and gamma). Moreover, we identified genes coding other urea processing enzymes including: cyanuric acid amidohydrolase, biuret amidohydrolase (BiuH), urea carboxylase and two copies of allophanate hydrolase (AtzF). The Paenibacillus genome encodes also one copy of ulilysin Peptidase_M43 (PF05572) with possible gelatinase function (Tallant et al., 2007), and 33 copies of Peptidase_M23 (PF01551), which includes mostly bacterial peptidoglycan hydrolases but also prokaryotic collagenases (Sasagawa et al., 1995).

Tree scale: 0.1

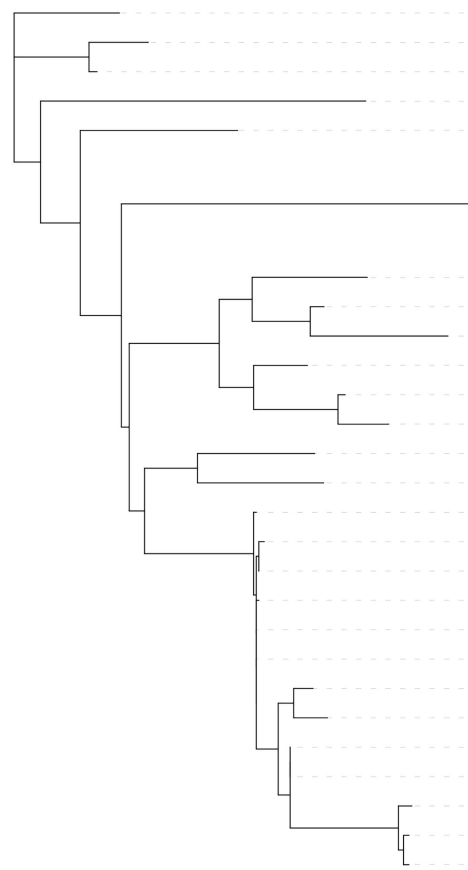

AB073203.1 Paenibacillus validus
AF130254.1 Paenibacillus koreensis
AY090110.1 Paenibacillus elgii
AB073362.1 Paenibacillus alginolyticus
AB073205.1 Paenibacillus larvae
GQ292772.1 Bacillus oceanisediminis
AB271747.1 Bacillus circulans
AY667496.1 Bacillus koreensis
Z84578.1 Bacillus cereus
AJ605292.1 Paenibacillus antarcticus
AJ011322.1 Paenibacillus borealis
AJ633647.1 Paenibacillus wynnii
AB073194.1 Paenibacillus chibensis
AB073188.1 Paenibacillus lautus
AB073189.1 Paenibacillus glucanolyticus
AB073196.1 Paenibacillus macerans
AJ320493.1 Paenibacillus polymyxa
FJ696708.1 Paenibacillus durus
MH777610.1 Paenibacillus illinoisensis
Paenibacillus sp.
NR 115624.1 Paenibacillus illinoisensis
NR 040884.1 Paenibacillus illinoisensis
AB073192.1 Paenibacillus illinoisensis
HQ258920.1 Paenibacillus xylanilyticus
KX350030.1 Paenibacillus xylanilyticus
AY427832.1 Paenibacillus xylanilyticus
NR 029109.1 Paenibacillus xylanilyticus
EU558284.1 Paenibacillus tundrae
EU558281.1 Paenibacillus xylanexedens
D85396.2 Paenibacillus amylolyticus

FIGURE 1 | Maximum likelihood phylogenetic tree of 16S rRNA shows the sequenced strain (bold font) groups with P. illinoisensis isolates. Branches with support values below 50 were collapsed, the tree was rooted with Bacillus sequences. 


\section{Genes Coding for CAZymes, Proteases and Transport-Related Proteins}

\section{Proteases}

The overall profile of encoded proteases is very similar in all sequenced fungi (Figure 2), with high numbers of encoded pepsin-like A01A peptidases, FtsH-like M41 peptidases, M48 peptidases active on di- and tripeptides, proteasome peptidases $\mathrm{T} 1$, lysosomal peptidases $\mathrm{C} 26$, and ubiquitin-specific proteases C19. These proteases, except for pepsin, contribute to intracellular protein turnover and regulation. Pepsin and subtilisin proteases are found in high copy numbers in all of the genomes pointing at a high degradation potential of these saprotrophic organisms. In all analyzed fungal genomes, we found an expansion of the C44 family. Proteins from this family are homologs of glutamine-fructose-6-phosphate transaminase (GFPT) and its precursor. GFTP is known to control the flux of glucose into the hexosamine pathway which plays a crucial role in the regulation of chitin synthesis in fungi (Maia, 1994). There is a two-fold expansion of S8A serine proteases in Mucorales when compared to Umbelopsidales (Supplementary Table 3). The same observation applies to family I4 which groups inhibitors of S8 peptidases. Umbelopsidales possess several families of cysteine peptidases (C15, C40, C110) and metalloproteases (M14A and M20A) absent from Mucorales genomes. The metalloproteases are likely involved in protein degradation whereas the cysteine peptidases have no obvious function in fungi.

Among sequenced Mucorales, the average number of proteincoding genes per peptidase family is elevated, which could be a consequence of the whole genome duplication (WGD) described in Mucor and Phycomyces by Corrochano and coworkers (Corrochano et al., 2016). However, BUSCO results show a low level of duplicated genes in all of the isolates. The genome of Thamnidium stands out as it encodes fewer proteases than remaining Mucorales. However, it may benefit from enzymes provided by its endohyphal bacterium which has genes encoding meat crumbling collagenase U32 and plenty of typically bacterial proteases representing families A36, A25, S15, S55, S66, S51, M29, and U57.

\section{Carbohydrate Active Enzymes}

All six genomes encode an extensive repertoire of carbohydrateactive enzymes (Figure 3). A high number of glycohydrolases is present exclusively in the genomes of Umbelopsis spp. and absent from all four Mucorales; these are GH1, GH2, lysozymes (GH24 and GH25), bacterial $\beta$-xylosidase GH52, endo- $\beta$-1,4-galactanase GH53, $\beta$-1,3-glucanase GH55, GH71, rhamnosidase GH78, $\alpha$-mannosidase GH92, exo- $\alpha-\mathrm{L}-1,5-$ arabinanase GH93, $\alpha$-L-fucosidase GH95 and peptidoglycan lytic transglycosylase (GH104), enzymes that hydrolyze fructosecontaining polysaccharides such as inulinases (GH32), endoglucanase like cellulase and endomannanase (GH5), phosphorylases (GH65), $\beta$-glucuronidases (GH79) and glucuronyl hydrolases (GH88) (Supplementary Table 4). All studied fungi have numerous copies of enzymes potentially involved in nutrient degradation and metabolite modification, for instance: $\alpha$-amylase (GH13), $\beta$-amylase (GH14), feruloyl esterase/acetyl xylan esterase (CE1), cholinesterase (CE10). All studied genomes have also an acetyl esterase that liberates acetic acid from xylo-oligomers (CE16). All also have homologs of GH13 $\alpha$-amylases, but only Umbelopsidales have representatives of the GH13_40 subfamily. This subfamily seems to group exclusively eukaryotic $\alpha$-amylases. Surprisingly, Umbelopsis spp. have also PL12 lyase, a bacterial enzyme used to access metazoan debris by specifically cleaving heparan sulfate-rich regions of acidic polysaccharides which are found in connective tissues (Su et al., 1996). Other typically bacterial enzymes found here are $\beta$-xylosidase GH52 and peptidoglycan lytic transglycosylase GH104. This repeated presence of bacterial enzymes may be a result of a horizontal gene transfer event from either endohyphal or free-living bacteria to Umbelopsidales or its ancestor. We also observed an expansion of GH16 hydrolases and hexosaminidase (GH20)

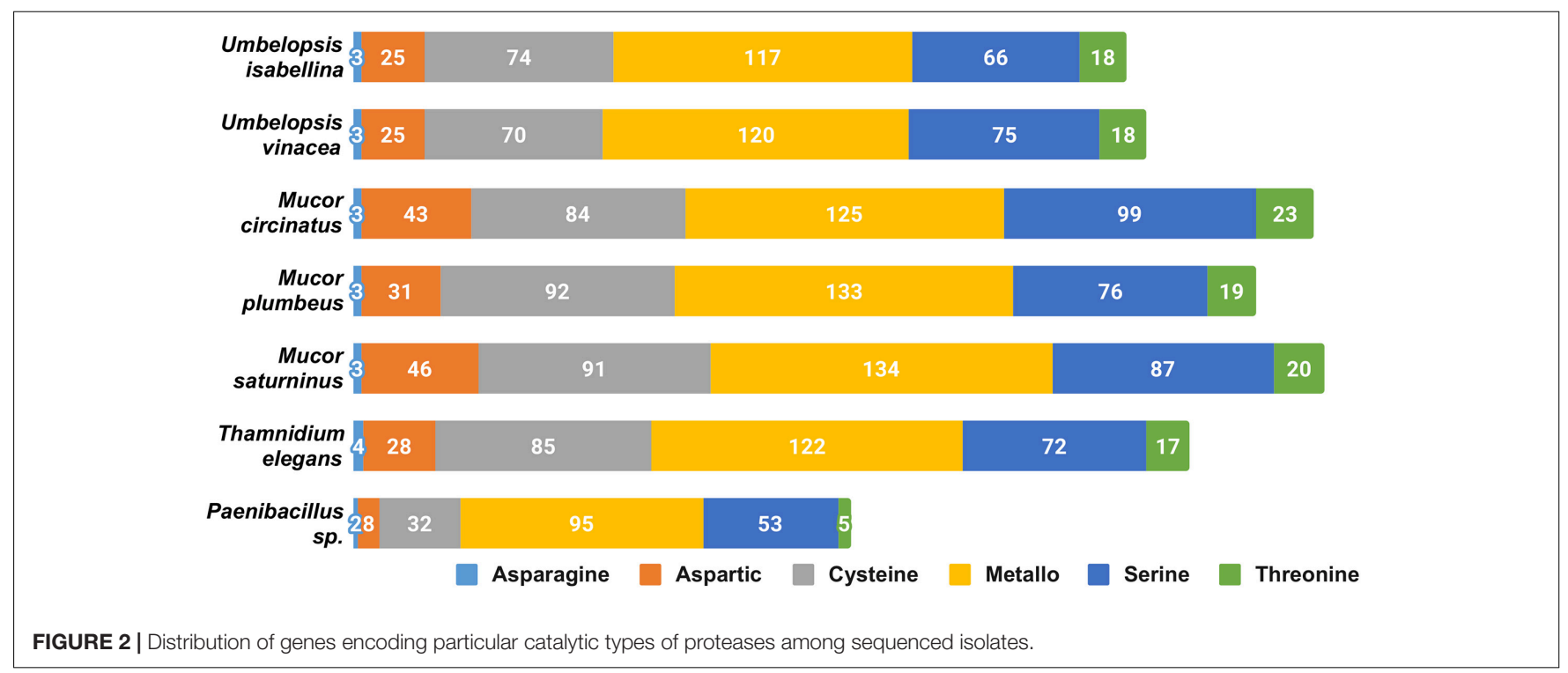




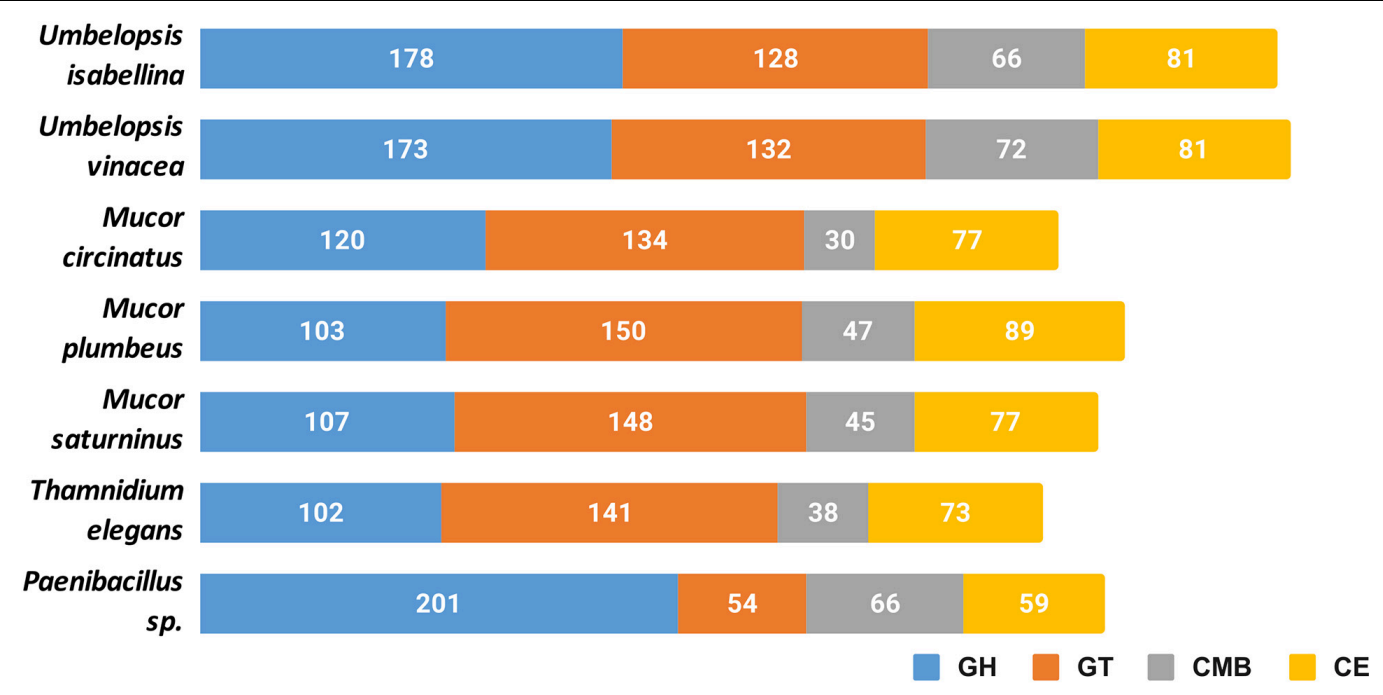

FIGURE 3 | Cazyme distribution in the sequenced genomes. GH, glycoside hydrolases; GT, glycosyltransferases; CMB, carbohydrate-binding modules; CE, carbohydrate esterase.

in Umbelopsidales compared to Mucorales. On the other hand, Umbelopsis spp. lack multiple enzymes belonging to several Cazy families, like acetyl xylan esterase (CE6), cellulosebinding modules (CBM6 and CMB9), chitin-binding module (CBM14), cell wall-related $\alpha$-1,6-mannanase (GH76) and endo- $\beta$-1,4-mannanase (GH134).

Noteworthy, all six fungi have expansions of protein families involved in cell wall remodeling. These include: chitooligosaccharide deacetylase (CE4), chitinase (GH18), chitin synthase (GT2), $\alpha$-1,2-mannosyltransferase (GT15), $\beta-1,3-\mathrm{N}$ acetylglucosaminyltransferase / $\beta$-1,3-glucuronyltransferase (GT49). Additionally, we found expansions of chitin or peptidoglycan (CBM50) binding modules and additional, broad range binding module (CBM13). Protein glycosylases are also particularly abundant in sequenced taxa and include homologs of Drosophila O-fut2 belonging to protein O- $\alpha$-fucosyltransferase (GT68) and glycoprotein $\alpha$-1,3-L-fucosyltransferase (GT10). The latter is encoded by 3 Mucor taxa and other known Mucoromycotina but not by Umbelopsis spp. Fucosylation may additionally mask Mucorales against animal immune systems, as fucose is widely present in the blood.

NagA $\quad \alpha$-N-acetylgalactosaminidase, NagZ $\quad \beta$-Nacetylhexosaminidase (GH109) involved in peptidoglycan recycling occur in multiple copies not only in Paenibacillus genome but also in sequenced fungal genomes. It is worth noting that Peanibacillus has three times more copies (30) of Nag genes than studied fungi. No proteins from this family had been characterized in Eukaryota.

Thamnidium elegans possesses the narrowest set of carbohydrate-processing enzymes which, like in the case of proteases, might be expanded by its associated bacterium. Paenibacillus encodes several unique enzymes, like pectate lyases (PL1, PL10, PL9) and rhamnogalacturonan (PL11), oligogalacturonate (PL22) and L-rhamnose- $\alpha$-1,4-D-glucuronate lyases (PL27). It also encodes lytic chitin monooxygenase (AA10) which may be either used to manipulate the host or to acquire nutrients. Additionally, Paenibacillus has expanded digestive repertoire including xylanases and xylosidases (GH11, GH30, GH43) and galactosidases (GH4, GH42, GH110). Interestingly, it encodes bacterial lipid-A-disaccharide synthase (GT19) and murein polymerase (GT51). Paenibacillus genome harbors 39 copies of S-layer homology (SLH) domain proteins which are important for biofilm formation (Janesch et al., 2013).

\section{Transporters}

Sequenced Mucorales have several classes of transporters: Amino Acid-Polyamine-Organocation (APC), GlycosidePentoside-Hexuronide (GPH), Putative Tripartite $\mathrm{Zn} 2+$ Transporter (TZT), ATP-binding Cassette (ABC), Major Facilitator Superfamily (MFS) and Drug/Metabolite Transporter (DMT) (Supplementary Table 5). Mucor circinatus possesses a huge expansion of MFS (347 compared to a median of 209 copies per genome). Thamnidium elegans has fewest transporter-encoding genes, but it is again possible that this is an effect of relationship with bacteria serving as a reservoir of necessary enzymes. Both Umbelopsis representatives have fewer transporters than analyzed Mucorales.

\section{Metabolic Clusters, Secondary Metabolites, and Cofactors}

The genome of Paenibacillus harbors NRPS, terpene, bacteriocin, T3PKS and S-layer-glycan producing clusters. Mucoromycotina were long considered devoid of secondary metabolite clusters. A review by Voigt et al. (2016) showed genetic determinants for natural product synthesis present in all analyzed genomes. Our newly sequenced genomes encode between 3 and 8 secondary metabolite clusters (according to AntiSMASH scans) belonging to different classes. Interestingly, all of the genomes encode terpene clusters (Supplementary Table 6) which potentially 
could produce new natural products like those isolated from Mortierella (Baldeweg et al., 2019).

Umbelopsidales additionally have a trans-2,3-dihydro-3hydroxyanthranilate isomerase $(\mathrm{PhzF})$ involved in phenazine biosynthesis with yet unknown biological product in fungi (Blankenfeldt et al., 2004). This gene is also present in other fungi and in Endogonales but seems to be missing in Mucorales. Umbelopsidales produce also a citronellol/citronellal dehydrogenase which converts citronellol to citronellic acid an odorous compound with antimicrobial properties. All four Mucorales isolates have a single gene coding for salicylate hydroxylase which is involved in plant host manipulation by Epichloë (Bastias et al., 2017) and other fungi.

All analyzed genomes have a B12 dependent methionine synthase homologous to the animal variant of the enzyme (PF02965, Met_synt_B12) and B12 independent synthase (PF01717, Meth_synt_2) encountered in Dikarya. Both enzymes have recently been found in other Mucoromycotina (Orlowska et al., 2020) and their presence suggests at least partial B12 dependence. Moreover, Umbelopsis spp. have methylmalonyl-CoA mutase (MUT), methylmalonylCoA/ethylmalonyl-CoA epimerase (MCEE), cob(I)alamin adenosyltransferase (MMAB, pduO) and ribonucleosidetriphosphate reductase (rtpR) - all using cobalamin. Mucorales have a B12-independent ribonucleotide reductase class II (nrdJ) and lack methylmalonyl-CoA mutase and MUT-epimerase genes.

\section{Sex Locus}

Sequenced isolates belong to heterothallic genera (Lee and Heitman, 2014) and genomic screening shows a single sex locus per genome. Generally, these loci contain a single high mobility group (HMG)-domain transcription factor gene (sexP or sexM), flanked by genes for an RNA helicase ( $r h A)$, a triosephosphate transporter $(t p t A)$ and an alginate lyase $(a g l)$ (Lee and Idnurm, 2017).

The sex locus of T. elegans and M. plumbeus is organized like in other Mucorales with genes in the following order agl/tptA/sex/rnhA (Lee and Idnurm, 2017). M. saturninus lacks the $t p t A$ gene and has a sex locus architecture $(\operatorname{agl} / \operatorname{sexP} / \mathrm{rnh} A)$ like M. mucedo (Wetzel et al., 2012). M. circinatus has remains of an integrase instead of the sex gene between $t p t A$ and $r n h A$ genes (agl/tptA/rve/rnhA). Mobile element insertions in the sex locus have already been documented in Phycomyces blakesleeanus (Idnurm et al., 2008).

The $r n h A$ gene seems to be excluded from the sex locus in both Umbelopsidales genomes, in which the sex locus is organized like in Mucorales but followed by a gene with an additional protein of unknown function belonging to DUF2405 (PF09435) family (agl/tptA/sexP/DUF2405). Schulz et al. (2016) described a similar architecture with the DUF2405 for Umbelopsis ramaniana from JGI database.

\section{Carbon Assimilation Profiles}

Carbon assimilation profiles obtained for six Mucoromycotina strains by screening on Biolog FF microplates are summarized in Supplementary Table 7 . None of the analyzed strains was able to use a full set of 95 tested carbon sources. Each strain was able to grow on 40 to 70 different substrates and had a unique carbon assimilation profile (Figure 4) with $T$. elegans being the most versatile degrader (Figure 5).

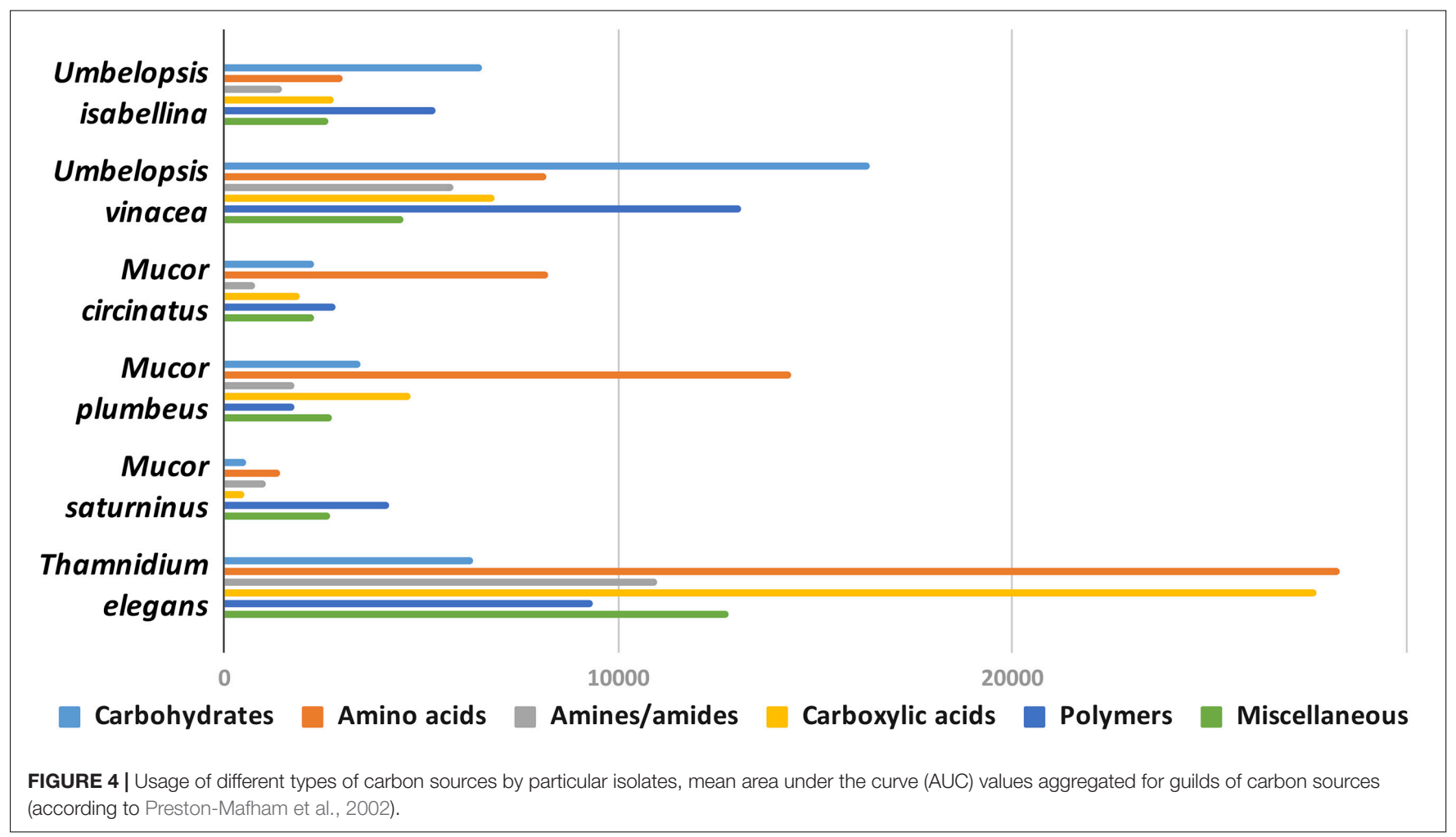




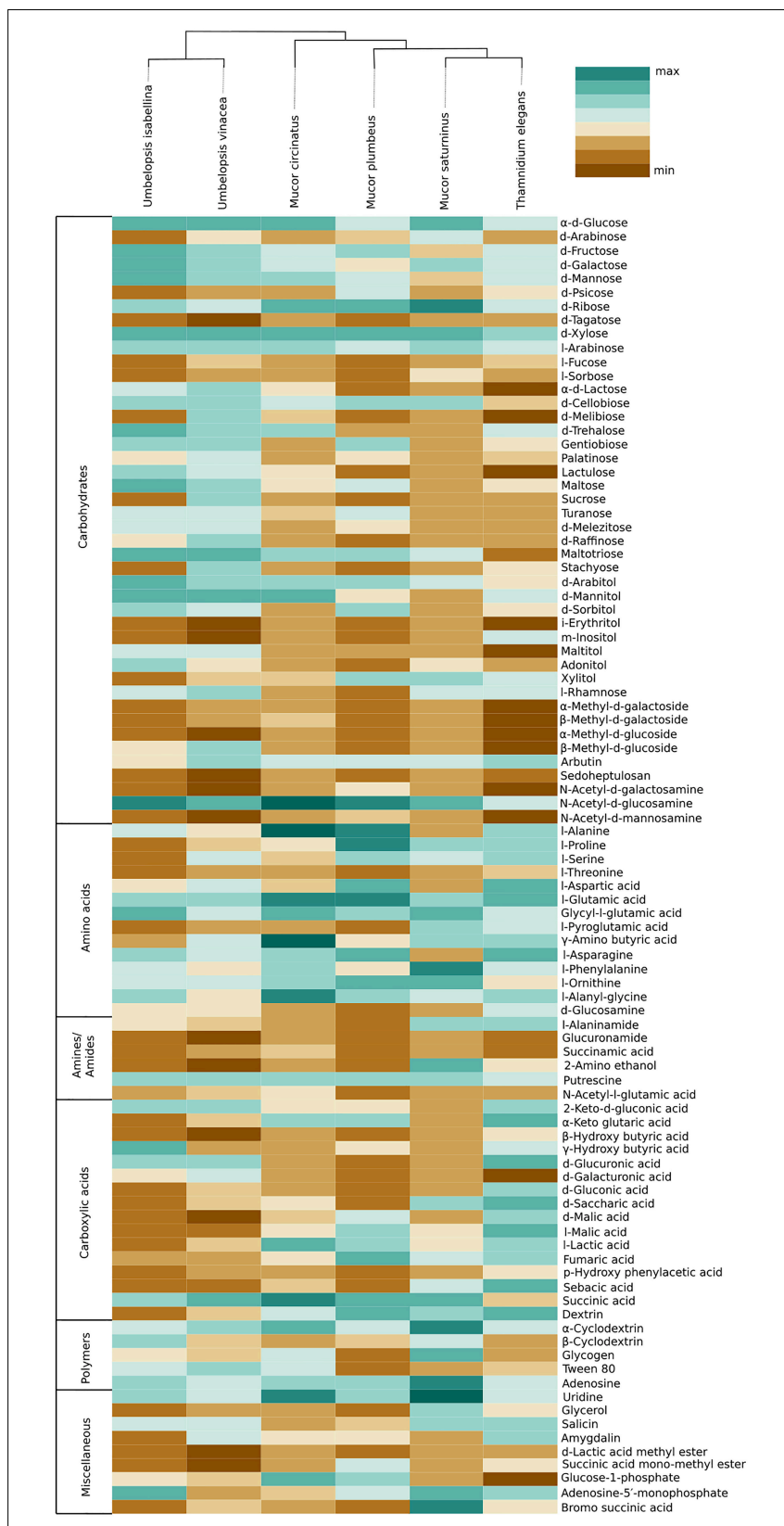

FIGURE 5 | Heatmap representing carbon source utilization capacity of 6 Mucoromycotina representatives obtained from Biolog FF MicroPlates (scale from brown - not used, to dark green - used very efficiently). The phylogenetic tree is illustrating evolutionary relationships of tested fungal strains. Tested carbon sources are grouped into guilds according to Preston-Mafham et al. (2002).

Umbelopsis spp. were more efficient in the utilization of carbohydrates, while Mucorales representatives showed the fastest growth rate on amino acids. Umbelopsis fungi were able to utilize Adonitol, d-Galacturonic Acid, Maltitol, $\beta$-Methyl-D-Glucoside and d-Raffinose whereas none of the four Mucorales grew on these substrates. Additionally, similarly to Mortierella elongata AG77 (Uehling et al., 2017), they represented elevated growth rate on other carbohydrates, like d-Galactose,
d-Mannose, 1-Arabinose, l-Rhamnose, d-Trehalose and lipid (Tween 80), which is consistent with predicted gene models (see the chapter on CaZymes - the presence of rhamnosidase GH78, $\alpha$-mannosidase GH92, exo- $\alpha$-L-1,5-arabinanase GH93, $\alpha$-L-fucosidase GH95). All four Mucor species can utilize D-Malic Acid and L-Malic Acid while these substrates seem inaccessible for Umbelopsis spp., which have only 3 copies of lactate dehydrogenase whereas Mucor spp. have from four to six enzymes from this family. Only $T$. elegans was able to utilize m-Inositol, Sedoheptulosan, $\beta$-Hydroxy-butyric Acid, p-Hydroxyphenyl-acetic Acid and L-Threonine. Previous reports showed efficient lactose assimilation by $T$. elegans what was not replicated in this study (Vamvakaki et al., 2010)Additionally, Thamnidium is distinguished by very efficient development on carboxylic acids. This ability may be explained by the presence of endobacteria whose proteome harbors representatives of M14 and M20 carboxypeptidase families. M14A is present uniquely in both Umbelopsis genomes while M14C occurs only in Paenibacillus. Also, peptidase T (M20B) is present exclusively in Paenibacillus, whereas fungal genomes have several copies of the remaining M20 subfamilies. Some of the analyzed compounds are available only to Paenibacillus, based on genomic evidence, e.g., 3-oxoacid CoA-transferase missing from sequenced Mucorales is present in Dikarya and bacteria, including Paenibacillus.

\section{Fungal Lipids}

For all six Mucorales, we determined the composition of sterols, fatty acids and phospholipids (Supplementary Tables 8-10). The presence of ergosterol, an important component of fungal plasma membranes, contributing to their stability, was confirmed in all 6 fungal biomasses.

Analyzed strains contained the following fatty acids: saturated $(14: 0,16: 0,18: 0)$, monounsaturated $(16: 1,18: 1)$, and polyunsaturated (18:2, 18:3) (Figure 6 and Supplementary Table 8). The lowest levels were observed for myristic (14:0) and palmitoleic (16:1) acids. A high percentage of oleic acid (18:1) is characteristic for the Umbelopsis strains and has also been reported by others (Fakas et al., 2009; Demir et al., 2013; Bernat et al., 2018). It is also the dominant fatty acid for all isolates except for Thamnidium elegans. A higher share of linolenic acid (18:3) was observed in Mucorales $18.5 \%$ compared to an average of $7.5 \%$ in Umbelopsidales. However, the efficiency of the production of particular fatty acids depends, inter alia, on the type of substrate used (Fakas et al., 2007). Among examined strains, $T$. elegans profile of fatty acids showed the lowest unsaturation index with the domination of palmitic acid $(16: 0,31.7 \%)$ and the highest share of stearic acid (18:0, 12\%), which may suggest increased membrane stiffness and its lower fluidity. Previous studies reported a higher unsaturation index with $55-70 \%$ of unsaturated FA w/w in total lipids and massive amounts of oleic acid inside the fungal lipids (Vamvakaki et al., 2010). Paradoxically, T. elegans has more desaturases than the remaining five isolates (6 compared to 4). Production of unsaturated fatty acids involves specialized desaturases. The delta-6-fatty acid desaturase involved in the conversion of 18:2 to $18: 3$ fatty acid and delta-12-fatty acid desaturase processing 18:1 to $18: 2$ were experimentally characterized in diverse 


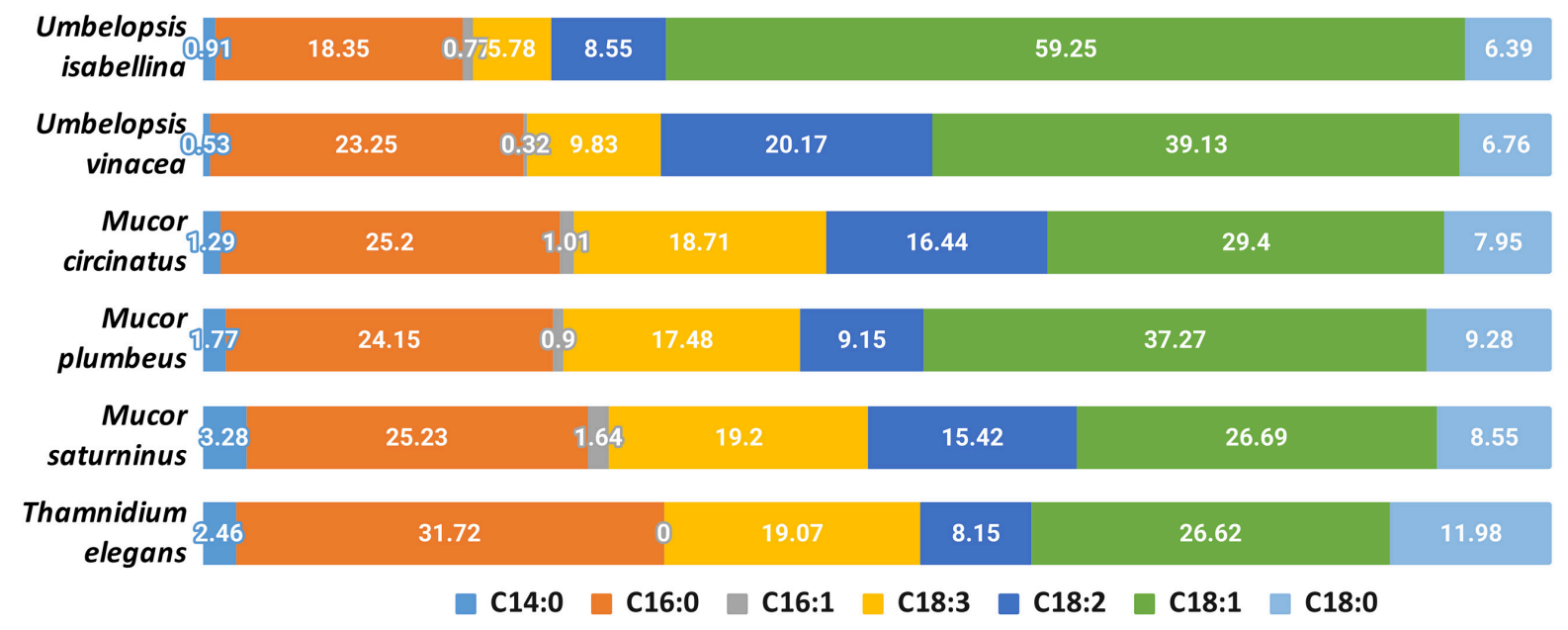

FIGURE 6 | Composition of fatty acids in the analyzed strains.

Mucoromycota including T. elegans, Mortierella alpina and Rhizopus oryzae (Huang et al., 1999; Wang et al., 2007; Zhu and Zhang, 2013). All isolates had both delta-6- and delta-12-fatty acid desaturases. T. elegans has four copies of delta-12-fatty acid desaturases with an N-terminal short accessory domain of the unknown function (PF11960, DUF3474) which often occurs with desaturase domains and a single delta- 6 desaturase with a Cytochrome b5-like Heme/Steroid binding domain (PF00173.28, Cyt-b5).

Using LC-MS/MS we determined the composition of phospholipids (PLs) present in the biomass of analyzed fungi (Figure 7, Supplementary Table 9). We identified 84 PLs species belonging to 6 classes: phosphatidic acid (PA), phosphatidylcholine (PC), phosphatidylethanolamine (PE), phosphatidylglycerol (PG), phosphatidylinositol (PI), and phosphatidylserine (PS). Sixty-two of them were chosen for subsequent quantitative assessments. This analysis of the PLs for selected strains revealed that PC and PE were predominant for selected Mucoromyotina and constituted up to $56 \%$ and $41 \%$ of the total cell PLs, respectively. PA, a lipid signal which usually constitutes a minute portion of PLs, in T. elegans and M. saturninus was found at $8 \%$ level. According to several reports, the increased levels of PA in living cells are a consequence of biotic and abiotic stress (Darwish et al., 2009; Bernat et al., 2014). The open question remains whether T. elegans and M. saturninus experienced stressing conditions during colony growth in the lab, or the observed increased level of PA is native for them as an adaptation to grow on animal substrate.

An important parameter describing the physical characteristics of biological membranes is $\mathrm{PC} / \mathrm{PE}$ ratio. $\mathrm{PC}$ has

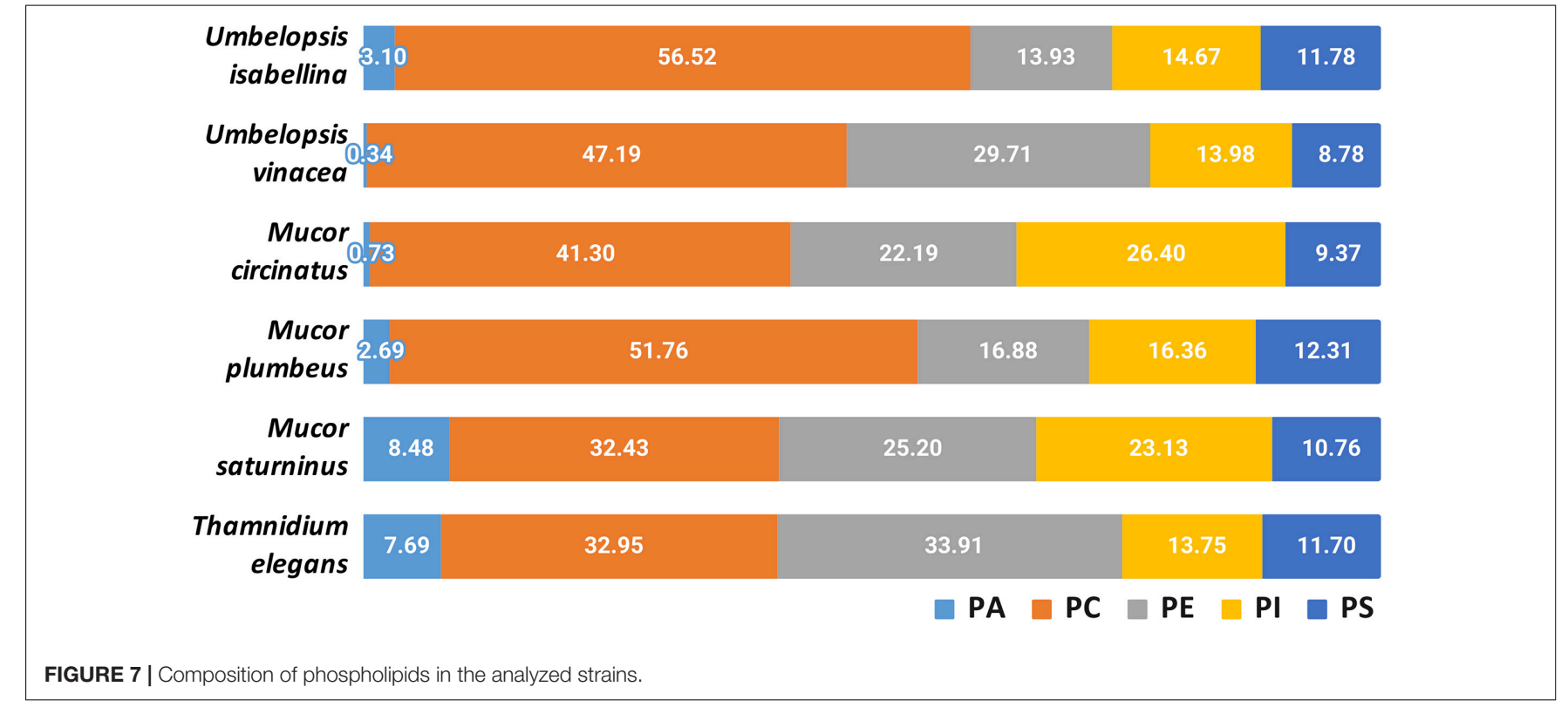


a bigger head group than PE. Tighter packing of PE and its acyl chains negatively influences fluidity of membranes as opposed to PC (Renne and de Kroon, 2018). PC can be synthesized from $\mathrm{PE}$, so they are closely related, and the balance between PE and $\mathrm{PC}$ is crucial for maintaining the physiological structure of the cell membrane. Among selected species, T. elegans showed the lowest PC/PE ratio (0.79), which may indicate a relatively greater stiffening of the membrane compared to other strains, which is consistent with lowered fatty acids unsaturation index.

Moreover, it is worth mentioning, that only T. elegans and U. vinacea had traces of PE 16:0 16:1 which is characteristic for diverse bacteria, including isolates of Pseudomonas putida, Bacillus subtilis or Escherichia coli (Gidden et al., 2009; Bernat et al., 2016, 2019). The dominant PLs species for all selected fungal strains were PC 18:2 18:3, PC 18:1 18:3, PC 18:1 18:1, PE 18:1 18:3, and PE 18:1 18:1. Similar results have already been reported for the PL profile of other Mucoromycotina representatives, Cunninghamella elegans (Bernat et al., 2014; Gardeli et al., 2017).

Other lipids which are important for the fungal metabolism are acylglycerols, triacylglycerols (TAGs) and diacylglycerols (DAGs) (Figure 8 and Supplementary Table 10). Yeasts store lipids mainly in the form of TAGs, and to less extent, DAGs (Lastovetsky et al., 2016). Triacyglycerols have been revealed, by far, as the most abundant lipid compound of Mucoromycotina (Fakas et al., 2007)Neutral lipids and triacylglycerols in particular constitute the main lipid fraction in U. isabellina and Cunninghamella echinulata (Fakas et al., 2007; Gardeli et al., 2017). In all six fungal strains, both acylglycerols were found. All analyzed strains accumulated more TAGs than DAGs but in T. elegans this ratio was significantly shifted toward 3:1 TAG to DAG, in contrast to the 5:1 in M. plumbeus or even 99:1 in M. saturninus. Since TAG synthesis in fungi requires $\mathrm{PA}$ and DAG, both present in T. elegans, observed DAG/TAG ratio might be a consequence of inhibition of acyl-CoA-dependent diacylglycerol acyl-transferase (DGA1) (Markgraf et al., 2014). Moreover, it seems that the acylglycerols were less enriched in polyunsaturated fatty acids, especially
18:3, than phospholipids. Unsaturation index of fatty acids for phospholipid class was higher than for total and neutral lipids for different Mucoromycotina species (C. echinulata, T. elegans, $U$. isabellina, Mucor sp.) tested by others researchers (Fakas et al., 2007; Vamvakaki et al., 2010; Gardeli et al., 2017)with $\gamma$-linolenic acid found in higher quantities in the PLs class.

Lastovetsky and co-workers (Lastovetsky et al., 2016) reported that $\mathrm{PE} / \mathrm{TAG}$ ratio plays a crucial role in establishing and maintaining symbiosis of the fungus Rhizopus microsporus (Mucoromycotina) and its Mycetohabitans endobacteria. $\mathrm{PE} / \mathrm{TAG}$ ratio close to $1: 1$ is characteristic for symbiosis and departure from that balance might shift the interaction toward antagonism. Interestingly, it was observed that among six strains, the PE/TAG ratio for T. elegans was closer to 1:1 (2.21) compared to the average (6.4) for all tested fungi. Analyzed strains all contain several copies of diacylglycerol kinase DGK genes (3-5) which is deemed responsible for maintaining the balance between TAG and PE.

The genome of Paenibacillus sp. encodes processive diacylglycerol beta-glucosyltransferase required for the synthesis of beta-diglucosyl-DAG - a predominant glycolipid found in Bacillales (Jorasch et al., 1998), as well as the Ugp snglycerol-3-phosphate transport system which transports glycerol-3-phosphate, essential for phospholipid biosynthesis.

\section{Cell-Wall Carbohydrates}

A quantitative analysis of the cell wall carbohydrates revealed the presence of high amounts of glucosamine and fucose, and low amounts of mannose, galactose, and glucose compared to an ascomycetous fungus Trichoderma reesei TU-6 (Figure 9). Glucosamine content in their cell wall was up to 10 fold higher compared to Trichoderma. High glucosamine fraction can be a highlight of the chitin-chitosan cell wall typical for Mucoromycotina (Bartnicki-Garcia, 1968).

Chitin together with glucans participates in the rigidity of the cell wall. The total content of glucosamine (representing chitin/chitosan fraction) and glucose (representing glucan fraction) in four Mucorales: M. plumbeus, M. saturninus,

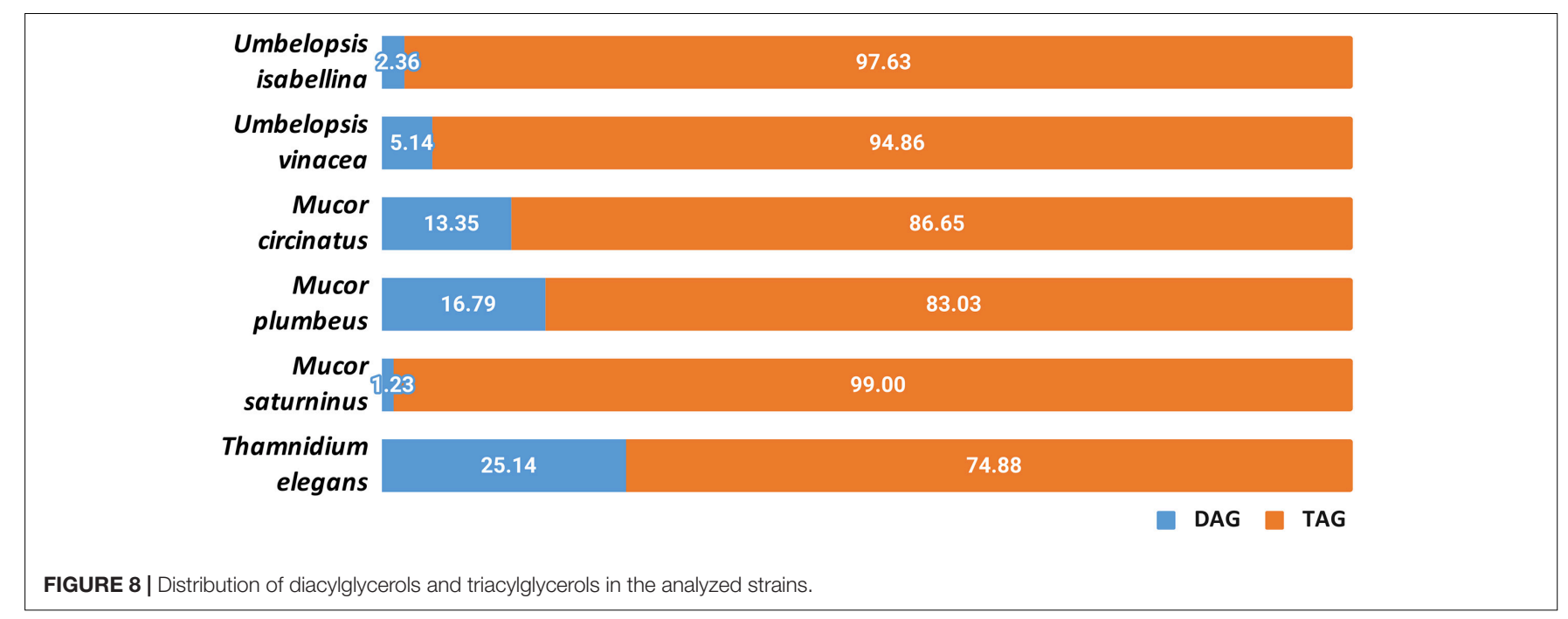




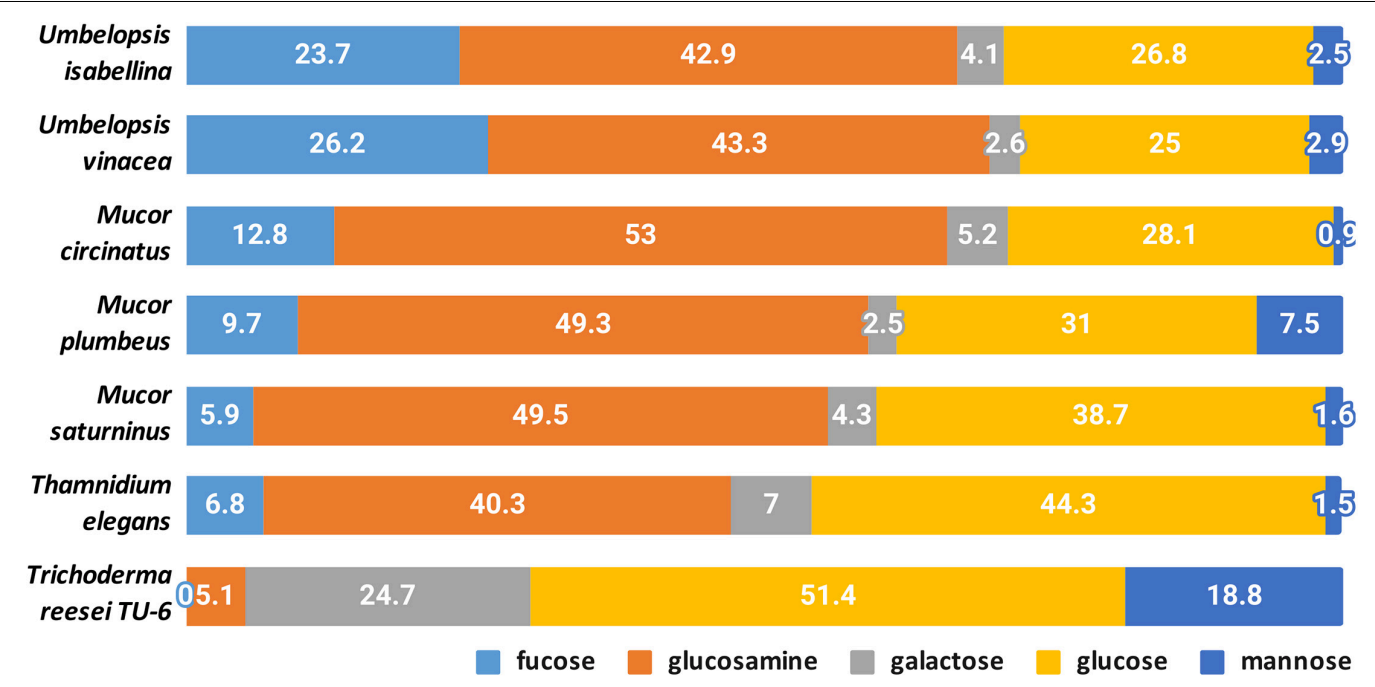

FIGURE 9 | Composition of cell-wall carbohydrates.

M. circinatus and T. elegans was above $80 \%$ of the cell wall carbohydrates. The amount of these two carbohydrates in Umbelopsis strains was lower and reached about 66 and $71 \%$ in $U$. isabelina and $U$. vinacea, respectively. Trichoderma had only $55 \%$ of these sugars in the cell wall and, in addition, glucose was the dominant one.

Melida and coworkers (Mélida et al., 2015) reported that genes encoding chitin biosynthesis (CHS) and its modification (CDA) were present in more copies in Phycomyces blakesleeanus and Rhizopus oryzae, compared to Neurospora crassa (Ascomycota), as well as fewer genes encoding glucan synthases. These results were confirmed in other studies on Mucorales (Lecointe et al., 2019) and our results show that respective CaZymes are abundant both in sequenced Umbelopsidales and Mucorales representatives. This could explain the possibility of synthesizing a huge amount of chitin in Mucoromycotina strains compared to Trichoderma.

Furthermore, all Mucoromycotina contain fucose, a carbohydrate characteristic for this subphylum, especially in such a high amount. Our study revealed that two Umbelopsis strains had from twice to three-fold more fucose when compared to $M$. circinatus and $M$. saturninus, respectively. Presence of fucan in the cell wall was previously reported for P. blakesleeanus and $R$. oryzae (Mélida et al., 2015). During the synthesis of fucan, fucose is transferred from GDP-fucose to polysaccharides by $\alpha$-fucosyltransferase and this enzyme was detected in the membrane fraction of $M$. circinelloides and partially characterized (Lecointe et al., 2019).

The $\alpha$-fucosyltransferase encoding genes were found in 2 and 4 copies in the genomes of $P$. blakesleeanus and $R$. oryzae, respectively, and they were not detected in N. crassa, which is in accordance with the fact that no fucose was detected in the cell wall of Neurospora (Mélida et al., 2015) and Trichoderma (this study). Differences in the copy number of $\alpha$-fucosyltransferase encoding genes do not explain the significant differences in the amount of fucose in the cell wall of Mucor spp. (4-6 copies) and
Umbelopsis spp. (3-4 copies). All six genomes have 1-3 copies of GDP-L-fucose synthase.

Thamnidium elegans despite the associated bacteria shows a cell wall carbohydrate profile similar to remaining Mucorales and other Mucoromycotina. This suggests that the interaction with a bacterial partner alters the metabolism, cell membrane composition but not the exoskeleton of the fungus.

\section{DISCUSSION}

Mucoromycotina representatives display a great diversity of genome sizes and genome complexity (Ma et al., 2009; Linde et al., 2014; Schwartze et al., 2014; Chibucos et al., 2015; Tang et al., 2015). Previously sequenced Umbelopsis genomes are very compact with 21-23 Mb and harbor around 9,100 genes (9,081 U. isabellina NBRC 7884 (Takeda et al., 2014), U. isabellina AD026: 9,193 genes (Nordberg et al., 2014) and U. ramanniana AG 9,931 genes (Nordberg et al., 2014). Known Endogonales genomes vary between $96 \mathrm{Mb}$ for Endogone sp. and $240 \mathrm{Mb}$ for Jimgerdemannia flammicorona and harbor from ca. 9,500 to 16,800 genes (Chang et al., 2019). Mucor and Rhizopus species, depending on the number of whole-genome duplications (WGD), have genomes ranging from $25 \mathrm{Mb}$ up to $55 \mathrm{Mb}$ and up to 17,000 genes (Nordberg et al., 2014; Corrochano et al., 2016; Gryganskyi et al., 2018).

In the present study, we aimed to point several differences between Umbelopsidales and Mucorales taxa. Besides limited genome size, Umbelopsis fungi have been demonstrated to be often associated with bacteria and to produce unsaturated fatty acids (Fakas et al., 2009). Our results build on top of these observations. Sequenced Umbelopsis taxa have compact genomes, almost devoid of repetitive sequences, and contain a moderate number of genes. They have a relatively high number of metabolism-related enzymes, especially glycohydrolases compared to Mucorales, but 
fewer peptidases. Moreover, we showed that all analyzed Mucoromycotina display comparable enzymatic capabilities tested on Biolog FF microplates and predicted from genomic data regardless of their genome size. Mucorales and Umbelopsidales representatives are mostly soil-inhabiting saprotrophs and require a broad spectrum of secreted enzymes which is reflected in high count of encoded peptidases, glycohydrolases and transporters. Nevertheless, they differ in the ability to use particular carbon sources, produce specific proteases, and have different ratios of different classes of phospholipids.

On one hand, Umbelopsis spp. in general have fewer secreted peptidases like pepsins and sublitisins than Mucorales. On the other, they encode several families of metalloproteases and cysteine proteases with unknown functions which are absent from Mucorales. The compact genomes of Umbelopsidales are richer in glycohydrolases and CaZymes. In consequence, they have a tendency to use carbohydrates efficiently and grow fast on these carbon sources. When compared to Mucorales, they produce higher amounts of 18:1 fatty acid and have more fucose in their cell wall. However, the meaning of these findings remains to be understood. Despite sharing an ecological niche, these two orders differ in genome size, associated bacteria and degrading capabilities but, nonetheless, share clear synapomorphic traits. Our experimental results support the validity of 18:3 lipids as a chemotaxonomic marker of Mucoromycotina and fucose as a specific component of their cell wall (Mélida et al., 2015; Lecointe et al., 2019).

Previous genomic studies covered diverse Mucorales representatives (Ma et al., 2009; Tang et al., 2015; Lebreton et al., 2020) whereas Umbelopsidales genomes were published in the form of brief genomic reports (Takeda et al., 2014) without a description of the genomic content. In this study, we aimed to fill this knowledge gap by bringing together genomic analyses with phenotype and biochemical studies, especially in the context of how these fungi function in their environment. Vast carbohydrate related enzyme repertoire observed in Umbelopsidales can be related to their ecology. According to the GlobalFungi database (Větrovský et al., 2020) the representatives of the genus Umbelopsis are present in 6155 out of 20009 global amplicon samples (ca 30\%). They are detected mainly in Europe, North America, and Australia, most often in soil, root, and shoots probes from forest or grassland biomes. The representatives of this genus are well-known late wood colonizers, that probably feed on the substrates which were decomposed by other organisms able to degrade complex substrates like cellulose (Richardson, 2009). However, Umbelopsis representatives are also often isolated from living plant material or forest soil (Sheng et al., 2019) and are considered to be plant growth-promoting organisms and root endophytes (Tejesvi et al., 2013; Huang et al., 2015). They can alter plant metabolism leading to the enhanced production of complex metabolites which are not produced without the endophyte (Qin et al., 2018). $U$. isabellina and $U$. vinacea described in this study also encoded metabolic clusters including terpenoid clusters.

The representatives of Umbelopsidales were also shown to represent relatively high resistance to some heavy metals like
Zn, Mn, Ni or Pb (Janicki et al., 2018) and xenobiotics, such as herbicides (Nykiel-Szymańska et al., 2018). The resistance may be correlated with the presence of numerous $\mathrm{ABC}$ transporters encoded in all analyzed genomes. Moreover, these genomes have a single arsenite resistance protein homologous to Absidia repens BCR42DRAFT_142507 and consisting of ARS2 (PF04959), DUF4187 (PF13821), RRM_1 (PF00076), SERRATE_Ars2_N (PF12066). Such domain architecture is conserved from chytrids to Entomophtoromycotina and Glomeromycotina. Interestingly, the genomes had neither Dikarya-type nor animal metallothioneins.

Umbelopsidales have also been detected in the deep-sea sediments from Magellan seamounts constituting 3.8\% of all OTUs (Yang et al., 2020). Although this finding is surprising as Umbelopsis representatives are well known terrestrial organisms, their presence may be explained by association with plant material. Interestingly, $0.85 \%$ of all amplicon samples in which Umbelopsis spp. were detected according to GlobalFungi database (Větrovský et al., 2020), are also originating from marine biome. The understanding of this pattern needs further research.

Some representatives of Umbelopsidales are recently considered as effective single cell oils (SCOs) producers as they are capable of producing high amounts of lipids (75\% to $84 \%$ in dry cell weight; w/w), including polyunsaturated fatty acids (PUFAs). These substances of high dietary and pharmaceutical importance are also considered as precursors for the synthesis of lipid-based biofuels. Although several Mucoromycota representatives were reported to synthesize PUFAs, $U$. isabellina cultivated on glucose has presented exceptionally high lipid production (comparable to the highest values achieved for genetically engineered SCO-producing bacterial strains) (Papanikolaou and Aggelis, 2019). In opposition to previous experiments (Gardeli et al., 2017), in our study the $U$. isabelina strain WA67209 growth on glucose was not more efficient than on xylose, and it was extensively assimilating neither glycerol, sucrose nor xylitol. Carbohydrate metabolism is closely related to metabolism of lipids (Dubois-Brissonnet et al., 2016) and lipid production depends on growth phase, their remodeling and their interplay with the synthesis of cellular polysaccharides. However, analyzed strains displayed a typical composition of PUFAs with a predominance of oleic acid (18:1), and higher levels of gamma-linolenic acid (18:3) in Mucorales compared to Umbelopsidales. Surprisingly, Thamnidium mycelium showed a relatively high saturation index possibly due to interaction with Paenibacillus. Bacteria produce more saturated fatty acids during biofilm formation yet it remains to be elucidated if the bacteria-fungus interaction has a similar effect on fatty acid composition in both partners (Dubois-Brissonnet et al., 2016). Thamnidium genome contains a high number of lipid metabolism-related genes and there is no clear explanation for the high level of fatty acid saturation. This phenotype was particularly unexpected since $T$. elegans has been used for biotechnological production of gamma-linoleic acid on diverse substrates especially in low temperature (Stredansky et al., 2000; Liu and Jin, 2008; Zikou et al., 2013). Similarly, the carbohydrate assimilation profiles of the analyzed 
strain WA18081 differed from other studies on this species (Papanikolaou et al., 2010; Pawłowska et al., 2019). It may be hypothesized that an extended set of lipid-processing enzymes in Thamnidium is required in order to balance the bacterial impact on lipid homeostasis and shift it back toward fungal characteristics.

Interestingly, several representatives of Umbelopsidales have recently been shown to be colonized by EHB from Burkholderiaceae (Okrasińska et al., 2021). Moreover, the metagenomic analysis of another oleaginous fungus - Mortierella elongata and its endosymbiont Mycoavidus cysteinexigens showed that bacteria alters the metabolism of the fatty acids of the host. Endosymbiont was shown not only to cause declines in the storage of carbohydrates, organic acids and nitrogenous metabolites but also to be involved in the catabolism of fungal fatty acids and changes volatile compounds profiles of the fungus (Uehling et al., 2017). Among six sequenced Mucoromycotina, we found numerous bacterial reads which assembled into a complete genome only in Thamnidium elegans. The presence of an associated bacteria is reflected in, among else, DAG/TAG lipids composition and utilization of carbohydrates which are accessible exclusively to Dikarya and bacteria. This phenomenon together with a limited set of carbohydrate-processing enzymes present in Thamnidium excludes the possibility of bacterial contamination and supports the hypothesis of intimate interaction with detected Paenibacillus. Further studies are needed to elucidate the molecular basis of the interaction and identify the predisposing features of the bacterial and fungal partners.

Most of the initial reports on intracellular fungal bacteria were based on microscopic observation of uncultivable bacteria inside fungal hyphae (Macdonald and Chandler, 1981). Nowadays, endohyphal bacteria (EHB) can be efficiently identified using genome sequencing methods. The traces of bacterial presence have been found in other published genomes of basal fungi (Naranjo Ortiz and Others, 2019). Identification of EHB can expand our scarce knowledge on the frequency and host range of fungal-bacteria interactions. Our finding of Paenibacillus sp. associated with Thamnidium elegans is in line with this trend. Diverse Bacillus bacteria were found living with truffles (Barbieri et al., 2005; Perlińska-Lenart et al., 2020). Paenibacillus has been reported from Laccaria bicolor (Bertaux et al., 2003), Sebacina vermifera (Sharma et al., 2008), and is known to produce pre-symbiotic and symbiotic interactions with Glomus (Bidondo et al., 2011).

The genus Paenibacillus was erected from Bacillus by Ash et al. (1993); it belongs to the family Paenibacillaceae and comprises 253 highly variable species ${ }^{1}$. The representatives of this genus were isolated from a wide range of sources of plant and animal origin. Paenibacillus tend to occupy a similar niche to Mucoromycotina molds as they inhabit soil and dung. The best-studied species - P. larvae is known to cause lethal disease of honeybees. However, some other species are known for their plant growth promotion capacities (via siderophores or phytohormones synthesis), others produce a variety of

${ }^{1}$ https://lpsn.dsmz.de/genus/paenibacillus antimicrobials and insecticides. Bacteria from this genus were also shown to produce a plethora of enzymes, like amylases, cellulases, hemicellulases, lipases, pectinases, oxygenases or dehydrogenases (Grady et al., 2016). The proteome of P. larvae includes a wide range of virulence factors including proteases and toxins (Erban et al., 2019). Paenibacillus validus stimulated the growth of Glomus intraradices (Hildebrandt et al., 2006) and P. vortex facilitates dispersal of Aspergillus fumigatus (Ingham et al., 2011). Although the representatives of Paenibacillus are known to promote plant growth and secrete several antimicrobial compounds, the endohyphal strain from our study did not encode any antibiotic compounds except for rifamycininactivating phosphotransferase. Rather, it provided multiple enzymes that significantly expanded the digestive capabilities of Thamnidium while reducing its genome size. Observed genome shrinking cannot be explained solely by the fragmented assembly of Thamnidium (its incompleteness is estimated at approximately $5 \%$ ) because the differences in CaZyme and peptidase abundance from remaining isolates are far greater.

One of the major differences between Thamnidium and the remaining isolates is in the lipid composition potentially contributing to cell membrane stiffness. EHB are known to influence host lipid production and bias the TAG/PE ratio (Lastovetsky et al., 2016). Thamnidium had the lowest TAG/PE ratio among tested isolates which might be a sign of symbiotic interaction with Paenibacillus, yet the noted ratio was still far from the 1:1 "symbiotic equilibrium" described in Lastovetsky's report (Lastovetsky et al., 2016). It is not known whether the TAG/PE values estimated for symbiosis between Rhizopus microsporus and Mycetohabitans are valid also for other models. It is an open question of how intimate and stable is the interaction between $T$. elegans and Paenibacillus sp. The altered traits of Thamnidium elegans compared to remaining isolates regardless of their evolutionary distances could be explained by the presence of an associated bacterium classified to Paenibacillus. In contrast to lipids, the cell wall carbohydrate composition of $T$. elegans remained unchanged. What we observed is that Thamnidium has several metabolic parameters altered but its morphology remained unchanged compared to strains without detectable bacterial partners.

The ancestors of extant Mucoromycotina were present among the first land colonizers and had the ability to access decomposing material. Genome sequencing and phenotyping of Mucorales and Umbelopsidales enabled us to look at the differences of these two old lineages within Mucoromycotina. There are several differences, particularly in their carbon source preferences and encoded carbohydrate repertoire, which hints at subtle niche differentiation. Importantly, predicted digestive capabilities are in line with experimental validation. Early diverging Mucoromycotina representatives possess features characteristic of fungi including ergosterol present in the membranes and a cell wall made of chitin and beta-glucan. Additionally, all studied Mucoromycotina representatives produce 18:3 gamma-linoleic acid and encrust their cell wall with fucose, both of which traits can be a handy discriminant for marking their presence in environmental samples. 


\section{MATERIALS AND METHODS}

\section{Isolates}

Six non-pathogenic, common and soil-borne representatives of Mucorales and Umbelopsidales were chosen for sequencing. Two of them represented the Umbelopsis genus, isolated from forest soil in Warsaw (Poland). Other two taxa (i.e., $M$. circinatus and $M$. plumbeus) were also soil-derived isolates from both Americas but representing Mucorales order. Finally, two Mucorales representatives that are well known for their proteolytic activity (i.e., Mucor saturninus and Thamnidium elegans) were also selected (Table 2). Related organisms were tested for biotechnological usage and reference genomic information for some of them (e.g., U. isabellina) was already available. The species-level identification of all isolates was confirmed by sequencing of ITS rDNA fragments (according to the protocol proposed by Walther and co-workers (Walther et al., 2013)) prior to genome sequencing.

\section{Phenotypic Microarray Plates}

FF phenotypic microarray plates (Biolog Inc., United States) were used to test the capacity of 6 strains to grow on 95 different carbon sources. Carbon sources were grouped into guilds according to Preston-Mafham et al. (2002). All fungal strains were cultured on Potato Dextrose Agar for 7 days and further their swabbed spores were suspended in FF inoculation fluid (deficient amount of carbon sources) to produce a final optical density of $0.036 \mathrm{~A}$ at $590 \mathrm{~nm}$. Spores' suspensions were then inoculated on FF microplates and incubated in the aerobic SpectrostarNano universal plate reader (BMR Labtech, Germany) for $96 \mathrm{~h}$ at $20{ }^{\circ} \mathrm{C}$. The analysis of each strain was done in three replicates. The metabolic activity was measured kinetically by determining the colorimetric reduction of a tetrazolium dye. Colorimetric values for wells containing carbon substrates were blanked against the control well. The result was considered positive when a difference between the metabolic activities of the first and last day of incubation was observed in all three repetitions. The mean values and standard deviations of AUC (area under the curve) were calculated for each strain and each guild of carbon sources. The metabolic activity of each species on a particular substrate was represented as a heatmap of log-transformed mean AUC values. All analyses were performed in RStudio using packages ggplot2 and vegan v2.4.2).

TABLE 2 | Used strains.

\begin{tabular}{lclc}
\hline Species & Strain number & Country of isolation & Substrate \\
\hline Umbelopsis isabellina & WA0000067209 & Poland & Forest soil \\
Umbelopsis vinacea & WA0000051536 & Poland (Bielany forest) & Forest soil \\
Mucor circinatus & CBS 142.35 & Brazil & Soil \\
Mucor plumbeus & CBS 226.32 & Canada & Forest soil \\
Mucor saturninus & WA0000017839 & Poland (Warsaw) & Cheese \\
Thamnidium elegans & WA0000018081 & Poland (Truskaw) & Horse dung
\end{tabular}

\section{Lipids Analysis Extraction}

Lipids from fungal cultures of the stationary phase of growth were extracted according to the method proposed by Folch et al. (1957) with some modifications. The fungal biomass was filtered and $0.1 \mathrm{mg}$ was transferred into Eppendorf tubes containing glass beads, $0.66 \mathrm{~mL}$ of chloroform and $0.33 \mathrm{~mL}$ of methanol. The homogenization process using a ball mill (FastPrep) was carried out for $1 \mathrm{~min}$. The mixture was extracted for $2 \mathrm{~min}$. In order to facilitate the separation of two layers, $0.2 \mathrm{~mL}$ of $0.9 \%$ saline was added. The lower layer was collected and evaporated.

\section{Phospholipid Determination}

The polar lipids were measured using an Agilent 1200 HPLC system (Santa Clara, CA, United States) and a 4500 Q-TRAP mass spectrometer (Sciex, Framingham, MA, United States) with an ESI source. For the reversed-phase chromatographic analysis, $10 \mu \mathrm{L}$ of the lipid extract was injected onto a Kinetex C18 column $(50 \mathrm{~mm} \times 2.1 \mathrm{~mm}$, particle size: $5 \mu \mathrm{m}$; Phenomenex, Torrance, CA, United States). The mobile phase consisted of 5$\mathrm{mM}$ ammonium formate in water (A) and 5-mM ammonium formate in methanol (B). The solvent gradient was initiated at $70 \% \mathrm{~B}$, increased to $95 \% \mathrm{~B}$ over $1.25 \mathrm{~min}$, and maintained at $95 \%$ $\mathrm{B}$ for $6 \mathrm{~min}$ before returning to the initial solvent composition over $3 \mathrm{~min}$. The column temperature was maintained at 40 ${ }^{\circ} \mathrm{C}$, and the flow rate was $500 \mu \mathrm{L} \mathrm{min}{ }^{-1}$. The instrumental settings of mass spectrometer were as follows: spray voltage $4500 \mathrm{~V}$, curtain gas (CUR) 25, nebulizer gas (GS1) 50, turbo gas (GS2) 60, and ion source temperature of $600{ }^{\circ} \mathrm{C}$. The data analysis was performed with the Analyst ${ }^{\mathrm{TM}}$ v1.6.2 software (Sciex, Framingham, MA, United States).

Two approaches were applied to identify PLs: targeted and untargeted. The untargeted approach was performed with the precursor ion scanning (precursor for $\mathrm{m} / \mathrm{z} 153$ ) survey scan, triggering the EPI experiments. On the basis of the untargeted analysis, a comprehensive list of the multiple reaction monitoring (MRM) transitions was generated.

\section{Acylglycerols}

Diacylglycerols and TAGs analysis was undertaken by liquid chromatography coupled to mass spectrometry (LC-MS) with electrospray ionization (ESI) on an QTRAP 4500 (Sciex). A Kinetex C18 column (see phospholipids determination) and mobile phases consisting of water (A), a mixture of acetonitrile:isopropanol (5:2, v/v) (B) and $5 \mathrm{mM}$ ammonium formate with $0.1 \%$ formic acid were used. The solvent gradient was initiated at $35 \% \mathrm{~B}$, increased to $100 \% \mathrm{~B}$ over $4 \mathrm{~min}$, and maintained at $100 \% \mathrm{~B}$ for $11 \mathrm{~min}$ before returning to the initial solvent composition over $2 \mathrm{~min}$. The column temperature was maintained at $40{ }^{\circ} \mathrm{C}$, and the flow rate was $600 \mu \mathrm{L} \mathrm{min}{ }^{-1}$. The QTRAP 4500 was operated with positive ionization at an electrospray voltage of $5500 \mathrm{~V}$ and a targeted multiple reaction monitoring (MRM) approach containing transitions for known precursor/product mass-to-charge ratio. Under these conditions, the TAG ionize as ammonium adducts. 


\section{Fatty Acid Analysis}

A lipid sample was diluted in $1.5 \mathrm{~mL}$ of methanol and transferred to a screw-capped glass test tube. To the lipid solution, $0.2 \mathrm{~mL}$ of toluene and $0.3 \mathrm{~mL}$ of the $8.0 \% \mathrm{HCl}$ solution were added (Ichihara and Fukubayashi, 2010). The tube was vortexed and then incubated at $45{ }^{\circ} \mathrm{C}$ overnight. After cooling to room temperature, $1 \mathrm{~mL}$ of hexane and $1 \mathrm{~mL}$ of water were added for the extraction of fatty acid methyl esters (FAMEs). The tube was vortexed, and then, $0.3 \mathrm{~mL}$ of the hexane layer was moved to the chromatographic vial. $1.6 \mu \mathrm{L}$ of the extract samples were analyzed using gas chromatography.

A FAMEs analysis was performed with an Agilent Model 7890 gas chromatograph, equipped with a 5975C mass detector. The separation was carried out in the capillary column HP 5 MS methyl polysiloxane $(30 \mathrm{~m} \times 0.25 \mathrm{~mm}$ i.d. $\times 0.25 \mathrm{~mm}$ $\mathrm{ft})$. The column temperature was maintained at $60{ }^{\circ} \mathrm{C}$ for $3 \mathrm{~min}$, then increased to $212{ }^{\circ} \mathrm{C}$ at the rate of $6^{\circ} \mathrm{C} \mathrm{min}^{-1}$, followed by an increase to $245{ }^{\circ} \mathrm{C}$ at the rate of $2^{\circ} \mathrm{C} \mathrm{min}^{-1}$, and finally to $280{ }^{\circ} \mathrm{C}$ at the rate of $20^{\circ} \mathrm{C} \mathrm{min}{ }^{-1}$. The column temperature was maintained at $280^{\circ} \mathrm{C}$ for $10 \mathrm{~min}$. Helium was used as the carrier gas at the flow rate of $1 \mathrm{ml} \mathrm{min}{ }^{-1}$. The injection port temperature was $250{ }^{\circ} \mathrm{C}$. The split injection was employed. Fungal fatty acids were identified by comparison with the retention times of the authentic standards (Sigma, Supelco) and the results were expressed as a percentage of the total amount of fatty acids.

Sterol analysis was undertaken using the QTRAP 3200 (Sciex) mass spectrometer connected to a 1200 series HPLC system. A Kinetex C18 column (see phospholipids determination) was used. The solvents were: water and methanol, both containing $5 \mathrm{mM}$ ammonium formate. Analytes were eluted with the following gradient: $40 \%$ solvent B from 0 to $1 \mathrm{~min}, 100 \%$ solvent B from 1 to $4 \mathrm{~min}, 40 \%$ solvent B from 4.0 to $4.1 \mathrm{~min}, 40 \%$ solvent $\mathrm{B}$ from 4.1 to $6 \mathrm{~min}$ with the flow rate $0.8 \mathrm{ml} \mathrm{min}^{-1}$. The QTRAP instrument was set to the positive ion mode, with the atmospheric pressure chemical ionization (APCI) temperature of $550^{\circ} \mathrm{C}$.

\section{Cell Wall Preparation and Determination of Cell Wall Carbohydrates}

Fungi were cultivated in PDB medium, washed with $10 \mathrm{mM}$ Tris/ $\mathrm{HCl}, \mathrm{pH} 7.5$, suspended in the same buffer, disintegrated with $0.5 \mathrm{~mm}$ glass beads in the presence of a protease inhibitor cocktail (Sigma-Aldrich) and centrifuged at $1500 \times g$ for $10 \mathrm{~min}$. The resulting pellet containing cell walls was washed with icecold $1 \mathrm{M} \mathrm{NaCl}$ until the disappearance of absorbance at 260280 nm (Nemčovič and Farkaš, 2001).

The lyophilized cell wall was hydrolyzed $\mathrm{o} / \mathrm{n}$ in $4 \mathrm{M}$ trifluoroacetic acid (TFA) at $100^{\circ} \mathrm{C}$. After cooling on ice, samples were centrifuged at $17000 \times g$ for $5 \mathrm{~min}$ at $4^{\circ} \mathrm{C}$. The supernatant was dried under $\mathrm{N} 2$ and washed twice with pure methanol. After removing methanol with $\mathrm{N} 2$, the pellet was resuspended in Mili Q water and purified on a Millipore Filter Device $(0.45 \mu \mathrm{m}$ pores) by centrifugation at $16000 \times g$ for $4 \mathrm{~min}$. Samples were stored at $-20^{\circ} \mathrm{C}$. Monosaccharides were determined by highperformance anion-exchange chromatography using a Dionex ICS-3000 Ion Chromatography System with a Carbo Pac PA10 analytical column. Neutral sugars were eluted with $18 \mathrm{mM} \mathrm{NaOH}$ at $0.25 \mathrm{ml} / \mathrm{min}$ (Zdebska and Kościelak, 1999).

\section{Culture Conditions and DNA Extraction}

All fungal strains were cultured on $4 \%$ Potato Dextrose Agar for 7 days at $20^{\circ} \mathrm{C}$. Total genomic DNA was extracted from $30 \mathrm{mg}$ of fresh mycelium following a CTAB-based chloroform extraction protocol (Doyle, 1991) and cleaned-up following caesium chloride density gradient centrifugation method (Garber and Yoder, 1983). DNA quality and concentration were estimated by $1 \%$ agarose gel electrophoresis and NanoDrop ${ }^{\circledR}$ (Thermo Fisher Scientific). Quantification of purified DNA was performed on a Qubit Fluorometer. The identity of all strains was confirmed by the preliminary sequencing of the internal transcribed spacer (ITS rDNA) region and with standard morphological identification procedures.

\section{Sequencing}

DNA was sequenced at the High Throughput Sequencing Facility of UNC, Chapel Hill, NC, United States.

Whole-genome sequencing was accomplished using a hybrid approach, combining Illumina short-read data with PacBio longread data.

Total cellular DNA was sheared using a Covaris E220 sonicator to achieve fragments with an average size of $500 \mathrm{bp}$. Then libraries were prepared using the Kapa Hyper kit. Libraries were size selected for insert fragments around 500 base pairs using Pippin Prep automatic DNA size selection system (Sage Science). Libraries were analyzed and quantified using a LabChip GX automated electrophoresis system (Caliper) and pooled. The pools were sequenced on Illumina MiSeq sequencer pairedend sequencing $(2 \times 300$ cycles $)$ to obtain longer reads and on Illumina HiSeq sequencer $2500(2 \times 150$ cycles $)$ to obtain more coverage.

For the PacBio RSII data, ten microgram aliquots of genomic DNA were sheared in a Covaris g-TUBE to a target fragment size of $20 \mathrm{~kb}$ using the shearing conditions provided in the Covaris g-TUBE user manual. The protocol for preparing a $20 \mathrm{~kb}$ library (Pacific Biosciences Procedure and Checklist- $20 \mathrm{~kb}$ Template Preparation Using Blue Pippin ${ }^{\mathrm{TM}}$ Size-Selection system) was subsequently followed, using $5 \mu \mathrm{g}$ of purified, sheared DNA as starting material. Template concentration was calculated using the Qubit fluorometer and the average size was determined by BioAnalyzer trace analysis and served as input to the Annealing and Binding Calculator v.2.1.0.2 (Pacific Biosciences) to prepare SMRTbell-template annealing and polymerase-template binding reactions, as well as the final dilution of the polymerase-bound template complex for sample plate loading and spike-in of control DNA. The PacBio reads were filtered to a minimum read length of $100 \mathrm{bp}$ and a minimum read quality score of 0.85 .

\section{Assembly}

PacBio reads were processed with SMRT Analysis Software. Spades (v.3.10.1) (Antipov et al., 2015) was chosen as a slow but high-quality assembler (Abbas et al., 2014). An initial assembly of Illumina reads alone with Abyss (Simpson et al., 2009) produced very fragmented and incomplete assemblies. Quast (v.4.6.1) 
was used to select the best assembly (Gurevich et al., 2013). The completeness of genome assembly and gene annotation was assessed using BUSCO (v.3b) (Simão et al., 2015), using BUSCO's fungal dataset universal single-copy orthologs from OrthoDB9 as reference.

BlobTools2 (Laetsch and Blaxter, 2017) was used to partition the scaffolds, on the basis of read coverage, G/C content, and taxonomic affiliation. The bacterial and fungal reads were assembled separately.

\section{Gene Calling}

MAKER (v.2.31.8) (Campbell et al., 2014) was used as the main annotation pipeline for gene prediction and annotation. As part of MAKER, AUGUSTUS (3.3) (Hoff and Stanke, 2013), and GeneMark-ES fungal version (v.4.21) were used as $a b$ initio gene predictors. The $a b$ initio was trained for a couple of times within MAKER based on sequence similarity search via BLAST and sequence alignment via Exonerate (v.2.2) (Slater and Birney, 2005) with default parameter settings, using 100,082 EST and 273,458 protein sequences of Mucoromycotina obtained from NCBI in January 2018, together with Swiss-Prot reference proteins (downloaded 18th January 2018). Repeats were predicted with RepeatMasker (v.4.0.7) (Smit et al., 2015) together with Tandem Repeat Finder (TRF) (v.4.04) (Benson, 1999) and RMBlast (NCBI blast package of RepeatMasker), based on Repbase database 2017 edition (Bao et al., 2015) as described in Muszewska et al. (2017b).

Gene calling in Paenibacillus was performed with Prokka (Seemann, 2014), an antimicrobial component search was done in Abricate (Seemann, 2019). A whole genome-based taxonomic analysis was performed in Type (Strain) Genome Server (TYGS) (Meier-Kolthoff and Göker, 2019) on 2020-06-01.

\section{Protein Coding Gene Annotation}

Predicted protein-coding genes were mapped on Cazy with DBCan (Yin et al., 2012) modified like in Muszewska et al. (2017a), MEROPS protease evalue 1E-10 (Rawlings and Bateman, 2020), PFAM evalue 0.001 (El-Gebali et al., 2019), INTERPROScan 5.28-67 (v(Jones et al., 2014)), CDD evalue 1E-5 (Lu et al., 2020), AntiSMASH 4.0 (-transatpks_daclusterblast-subclusterblast-knownclusterblast-smcogs-cassisborderpredict-full-hmmer) (Blin et al., 2017), TCDB evalue 1E-5 (Saier et al., 2016). RNAi components were filtered with an evalue of 1E-10. KEGG mapping was performed with kofamscan 1.3.0 with default settings (Kanehisa et al., 2016).

\section{Phylogenetic Analysis of Bacteria Found in the Genome of Thamnidium}

16S rRNA sequence was extracted from the bacterial reads found in Thamnidium using blast. Then it was combined with publicly available $16 \mathrm{~S}$ sequences of several species and strains of Paenibacillus and Bacillus (GB accession numbers can be found on Figure 1). Sequences were then aligned using MAFFT (Katoh and Standley, 2013) and trimmed using trimal-automated 1 (Capella-Gutiérrez et al., 2009). Then the best evolution model was detected using modeltest-ng across all evolutionary models
(Darriba et al., 2020) (TrN+I+G4 model selected based on AIC, $\mathrm{BIC}$ and AICc criteria) and phylogenetic tree was calculated using raxml-ng (Kozlov et al., 2019) with 1000 bootstrap replicates. The tree was then rooted using four Bacillus sequences.

\section{DATA AVAILABILITY STATEMENT}

The datasets presented in this study can be found in online repositories. The names of the repository/repositories and accession number(s) can be found below: NCBI BioProject, accession no: PRJNA668042. Raw reads for all fungal strains are available in the SRA database under accession numbers SRR12875449-SRR12875464. Assemblies and annotations are deposited under accession numbers JAEPQZ000000000JAEPRE000000000 (Umbelopsisisabellina WA0000067209 under JAEPQZ000000000, Umbelopsisvinacea WA0000051536 under JAEPRA000000000, Mucorcircinatus CBS 142.35 under JAEPRB000000000, Mucorplumbeus CBS 226.32 under JAEPRC000000000, Mucorsaturninus WA0000017839 under JAEPRD000000000, and Thamnidiumelegans WA0000018081 under JAEPRE000000000). Paenibacillus reads can be found under accession number SRR13045247.

\section{AUTHOR CONTRIBUTIONS}

AM and JP designed the study. AM performed assembly, annotation, and sequence analyses. JK, PB, AO, KSt, JP, and AM interpreted the data and drafted the manuscript. KSt, JP, and AM wrote the manuscript. EM and PM performed wholegenome sequencing. OD, UZ, and SP performed experimental procedures and prepared the samples. TA-P and KSz performed carbon source usage experiments. UP-L and JK analyzed cell wall composition. PB analyzed lipid composition. JP analyzed carbon source usage. All authors contributed to the article and approved the submitted version.

\section{FUNDING}

This work was supported by the National Science Centre, Poland, under grants no 2012/07/D/NZ2/04286 and 2017/25/B/NZ2/01880 to AM, 2019/35/D/NZ2/03411 to KSt, and $2015 / 17 / \mathrm{D} / \mathrm{NZ8} / 00778$ and 2017/25/B/NZ8/00473 to JP.

\section{ACKNOWLEDGMENTS}

We thank Gustavo Henrique Goldman and Marcin Grynberg for their insight and comments about the manuscript.

\section{SUPPLEMENTARY MATERIAL}

The Supplementary Material for this article can be found online at: https://www.frontiersin.org/articles/10.3389/fmicb.2021. 636986/full\#supplementary-material 
Supplementary Figure 1 | Tree inferred with FastME 2.1.6.1 (Lefort et al., 2015) from Genome BLAST Distance Phylogeny approach distances calculated from genome sequences of Paenibacillus isolates (Meier-Kolthoff et al., 2013). The numbers above branches are pseudo-bootstrap support values $>60 \%$ from 100 replications, with average branch support of $47.8 \%$. The tree was rooted at the midpoint by the program. Pae_T is the Paenibacillus sequenced as associated with Thamnidium elegans groups with $P$. illinoisensis strains.

Supplementary Table 1 | Repetitive elements in analyzed genomes.

Supplementary Table 2 | RNAi pathway components in the analyzed genomes.

Supplementary Table 3 | Merops families of peptidases in analyzed genomes.

Supplementary Table 4 | CaZyme families in analyzed genomes.

\section{REFERENCES}

Abbas, M. M., Malluhi, Q. M., and Balakrishnan, P. (2014). Assessment of de novo assemblers for draft genomes: a case study with fungal genomes. BMC Genomics. 15:S10.

Antipov, D., Korobeynikov, A., McLean, J. S., and Pevzner, P. A. (2015). hybridSPAdes: an algorithm for hybrid assembly of short and long reads. Bioinformatics 32, 1009-1015. doi: 10.1093/bioinformatics/btv688

Ash, C., Priest, F. G., and Collins M. D. (1993). Molecular identification of rRNA group 3 bacilli (Ash, Farrow, Wallbanks and Collins) using a PCR probe test. Proposal for the creation of a new genus Paenibacillus. Anton. Van Leeuwenhoek. 64, 253-260. doi: 10.1007/bf00873085

Baldeweg, F., Warncke, P., Fischer, D., and Gressler, M. (2019). Fungal Biosurfactants from Mortierella alpina. Org. Lett. 21, 1444-1448. doi: 10.1021/ acs.orglett.9b00193

Bao, W., Kojima, K. K., and Kohany, O. (2015). Repbase Update, a database of repetitive elements in eukaryotic genomes. Mob DNA. 6:11.

Barbieri, E., Bertini, L., Rossi, I., Ceccaroli, P., Saltarelli, R., Guidi, C., et al. (2005). New evidence for bacterial diversity in the ascoma of the ectomycorrhizal fungus Tuber borchii Vittad. FEMS Microbiol. Lett. 247, 23-35.

Bartnicki-Garcia, S. (1968). Cell Wall Chemistry, Morphogenesis, and Taxonomy of Fungi. Annu. Rev. Microbiol. 22, 87-108. doi: 10.1146/annurev.mi.22.100168. 000511

Bastias, D. A., Alejandra Martínez-Ghersa, M., Ballaré, C. L., and Gundel, P. E. (2017). Epichloë Fungal Endophytes and Plant Defenses: not Just Alkaloids. Trends Plant Sci. 5, 939-948. doi: 10.1016/j.tplants.2017.08.005

Benson, G. (1999). Tandem repeats finder: a program to analyze DNA sequences. Nucleic Acids Res. 27, 573-580. doi: 10.1093/nar/27.2.573

Bernat, P., Gajewska, E., Szewczyk, R., Słaba, M., and Długoński, J. (2014). Tributyltin (TBT) induces oxidative stress and modifies lipid profile in the filamentous fungus Cunninghamella elegans. Environ. Sci. Pollut. Res. Int. 21, 4228-4235. doi: 10.1007/s11356-013-2375-5

Bernat, P., Nesme, J., Paraszkiewicz, K., Schloter, M., and Płaza, G. (2019). Characterization of Extracellular Biosurfactants Expressed by a Pseudomonas putida Strain Isolated from the Interior of Healthy Roots from Sida hermaphrodita Grown in a Heavy Metal Contaminated Soil. Curr. Microbiol. 19, 1320-1329. doi: 10.1007/s00284-019-01757-x

Bernat, P., Nykiel-Szymańska, J., Stolarek, P., Słaba, M., Szewczyk, R., Różalska, S. (2018). 2,4-dichlorophenoxyacetic acid-induced oxidative stress: metabolome and membrane modifications in Umbelopsis isabellina, a herbicide degrader. PLoS One. 13:e0199677. doi: 10.1371/journal.pone.0199677

Bernat, P., Paraszkiewicz, K., Siewiera, P., Moryl, M., Płaza, G., Chojniak, J. (2016). Lipid composition in a strain of Bacillus subtilis, a producer of iturin A lipopeptides that are active against uropathogenic bacteria. World J. Microbiol. Biotechnol. 2016:2126. doi: 10.1007/s11274-016-2126-0

Bertaux, J., Schmid, M., Prevost-Boure, N. C., Churin, J. L., Hartmann, A., Garbaye, J., et al. (2003). In situ identification of intracellular bacteria related to Paenibacillus spp. in the mycelium of the ectomycorrhizal fungus Laccaria bicolor S238N. Appl. Environ. Microbiol. 69, 4243-4248. doi: 10.1128/aem.69.7. 4243-4248.2003
Supplementary Table 5 | TCDB families of transporters in analyzed genomes.

Supplementary Table 6 | Antismash metabolic clusters in analyzed genomes.

Supplementary Table 7 | Carbon source usage of the isolates.

Supplementary Table $\mathbf{8}$ | Total lipid and fatty acid composition of the isolates.

Supplementary Table 9 | Phospholipid composition of the isolates.

Supplementary Table 10 | Diacylglycerols and triacylglycerols content in the analyzed isolates.

Supplementary Table 11 | Type (strain) genome server results for Paenibacillus sp. genome listing DDH scores for related organisms.

Bidartondo, M. I., Read, D. J., Trappe, J. M., Merckx, V., Ligrone, R., Duckett, J. G. (2011). The dawn of symbiosis between plants and fungi. Biol. Lett. 7, 574-577. doi: $10.1098 / \mathrm{rsbl} .2010 .1203$

Bidondo, L. F., Silvani, V., Colombo, R., Pérgola, M., Bompadre, J., Godeas, A. (2011). Pre-symbiotic and symbiotic interactions between Glomus intraradices and two Paenibacillus species isolated from AM propagules. In vitro and in vivo assays with soybean (AG043RG) as plant host. Soil Biol. Biochem. 2011, 1866-1872. doi: 10.1016/j.soilbio.2011.05.004

Blankenfeldt, W., Kuzin, A. P., Skarina, T., Korniyenko, Y., Tong, L., Bayer, P., et al. (2004). Structure and function of the phenazine biosynthetic protein PhzF from Pseudomonas fluorescens. Proc. Natl. Acad. Sci. U. S. A. 101, 16431-16436. doi: 10.1073/pnas.0407371101

Blin, K., Wolf, T., Chevrette, M. G., Lu, X., Schwalen, C. J., Kautsar, S. A., et al. (2017). antiSMASH 4.0-improvements in chemistry prediction and gene cluster boundary identification. Nucleic Acids Res. 45, W36-W41.

Bonfante P and Venice, F. (2020). Mucoromycota: going to the roots of plantinteracting fungi. Fungal Biol. Rev. 12, 100-113. doi: 10.1016/j.fbr.2019.12.003

Calo, S., Nicolás FE, Lee, S. C., Vila, A., Cervantes, M., Torres-Martinez, S., et al. (2017). A non-canonical RNA degradation pathway suppresses RNAidependent epimutations in the human fungal pathogen Mucor circinelloides. PLoS Genet. 13:e1006686. doi: 10.1371/journal.pgen.1006686

Campbell, M. S., Holt, C., Moore, B., and Yandell, M. (2014). Genome Annotation and Curation Using MAKER and MAKER-P. Curr. Prot. Bioinform. 48, 4.11.14.11.39.

Capella-Gutiérrez, S., Silla-Martínez, J. M., and Gabaldón, T. (2009). trimAl: a tool for automated alignment trimming in large-scale phylogenetic analyses. Bioinformatics 25, 1972-1973. doi: 10.1093/bioinformatics/btp348

Castanera, R., Pérez, G., López, L., Sancho, R., Santoyo, F., Alfaro, M., et al. (2014). Highly expressed captured genes and cross-kingdom domains present in Helitrons create novel diversity in Pleurotus ostreatus and other fungi. BMC Genom. 15:1071. doi: 10.1186/1471-2164-15-1071

Cervantes, M., Vila, A., Nicolás, F. E, Moxon, S., de Haro, J. P., Dalmay, T., et al. (2013). A single argonaute gene participates in exogenous and endogenous RNAi and controls cellular functions in the basal fungus Mucor circinelloides. PLoS One. 8:e69283. doi: 10.1371/journal.pone.0069283

Chang, Y., Desirò A, Na, H., Sandor, L., Lipzen, A., Clum, A., et al. (2019). Phylogenomics of Endogonaceae and evolution of mycorrhizas within Mucoromycota. New Phytol. 222, 511-525. doi: 10.1111/nph.15613

Chibucos, M. C., Etienne, K. A., Orvis, J., Lee, H., Daugherty, S., Lockhart, S. R., et al. (2015). The genome sequence of four isolates from the family Lichtheimiaceae. Pathog Dis. 73:ftv024. doi: 10.1093/femspd/ftv024

Cornely, O. A., Alastruey-Izquierdo, A., Arenz, D., Chen, S. C. A., Dannaoui, E., Hochhegger, B., et al. (2019). Global guideline for the diagnosis and management of mucormycosis: an initiative of the European Confederation of Medical Mycology in cooperation with the Mycoses Study Group Education and Research Consortium. Lancet Infect. Dis. 19, 405-421e. doi: 10.1016/s14733099(19)30312-3

Corrochano, L. M., Kuo, A., Marcet-Houben, M., Polaino, S., Salamov, A., Villalobos-Escobedo, J. M., et al. (2016). Expansion of Signal Transduction Pathways in Fungi by Extensive Genome Duplication. Curr. Biol. 26, 15771584 . 
da Silva, M., Manfio, G. P., and Canhos, V. P. (1998). Characterization of selected strains of mucorales using fatty acid profiles. Rev. Microbiol. 29, 276-281. doi: 10.1590/s0001-37141998000400008

Darriba, D., Posada, D., Kozlov, A. M., Stamatakis, A., Morel, B., Flouri, T. (2020). ModelTest-NG: a new and scalable tool for the selection of DNA and protein evolutionary models. Mol. Biol. Evol. 37, 291-294. doi: 10.1101/61 2903

Darwish, E., Testerink, C., Khalil, M., El-Shihy, O., and Munnik, T. (2009). Phospholipid signaling responses in salt-stressed rice leaves. Plant Cell Physiol. 50, 986-997. doi: 10.1093/pcp/pcp051

Dashdorj, D., Tripathi, V. K., Cho, S., Kim, Y., and Hwang, I. (2016). Dry aging of beef; Review. Hanguk Tongmul Chawon Kwahakh. Chi. 58:20.

Demir, M., Turhan, I., Kucukcetin, A., and Alpkent, Z. (2013). Oil production by Mortierella isabellina from whey treated with lactase. Bioresour Technol.;128: 365-369. doi: 10.1016/j.biortech.2012.10.078

Desirò, A, Salvioli, A., Ngonkeu, E. L., Mondo, S. J., Epis, S., Faccio, A., et al. (2014). Detection of a novel intracellular microbiome hosted in arbuscular mycorrhizal fungi. ISME J. 8, 257-270. doi: 10.1038/ismej.2013.151

Desirò, A., Hao, Z., Liber, J. A., Benucci GMN, Lowry, D., Roberson, R., et al. (2018). Mycoplasma-related endobacteria within Mortierellomycotina fungi: diversity, distribution and functional insights into their lifestyle. ISME J. 12, 1743-1757. doi: 10.1038/s41396-018-0053-9

Desirò, A., Rimington, W. R., Jacob, A., Pol, N. V., Smith, M. E., Trappe, J. M., et al. (2017). Multigene phylogeny of Endogonales, an early diverging lineage of fungi associated with plants. IMA Fungus. 8, 245-257. doi: 10.5598/imafungus. 2017.08.02.03

Doyle, J. (1991). DNA Protocols for Plants. Mole. Techniq. Taxon. 642, 283-293. doi: 10.1007/978-3-642-83962-7_18

Dubois-Brissonnet, F., Trotier, E., and Briandet, R. (2016). The Biofilm Lifestyle Involves an Increase in Bacterial Membrane Saturated Fatty Acids. Front. Microbiol. 7:1673.

El-Gebali, S., Mistry, J., Bateman, A., Eddy, S. R., Luciani, A., Potter, S. C., et al. (2019). The Pfam protein families database in 2019. Nucleic Acids Res. 47, D427-D432.

Erban, T., Zitek, J., Bodrinova, M., Talacko, P., Bartos, M., Hrabak, J. (2019). Comprehensive proteomic analysis of exoproteins expressed by ERIC, I., II, III and IV Paenibacillus larvae genotypes reveals a wide range of virulence factors. Virulence 363-375. doi: 10.1080/21505594.2019.1603133

Fakas, S., Galiotou-Panayotou, M., Papanikolaou, S., Komaitis, M., and Aggelis, G. (2007). Compositional shifts in lipid fractions during lipid turnover in Cunninghamella echinulata. Enzyme Microbial. Technol. 2007, 1321-1327. doi: 10.1016/j.enzmictec.2006.10.005

Fakas, S., Makri, A., Mavromati, M., Tselepi, M., and Aggelis, G. (2009). Fatty acid composition in lipid fractions lengthwise the mycelium of Mortierella isabellina and lipid production by solid state fermentation. Bioresour. Technol. 100, 6118-6120. doi: 10.1016/j.biortech.2009.06.015

Field, K. J., Rimington, W. R., Bidartondo, M. I., Allinson, K. E., Beerling, D. J., Cameron, D. D., et al. (2015). First evidence of mutualism between ancient plant lineages (Haplomitriopsida liverworts) and Mucoromycotina fungi and its response to simulated Palaeozoic changes in atmospheric CO2. New Phytol. 205, 743-756. doi: 10.1111/nph.13024

Folch, J., Lees, M., and Sloane Stanley, G. H. (1957). A simple method for the isolation and purification of total lipides from animal tissues. J. Biol. Chem. 226, 497-509. doi: 10.1016/s0021-9258(18)64849-5

Garber, R. C., and Yoder, O. C. (1983). Isolation of DNA from filamentous fungi and separation into nuclear, mitochondrial, ribosomal, and plasmid components. Anal. Biochem. 135, 416-422. doi: 10.1016/0003-2697(83)90704-2

Gardeli, C., Athenaki, M., Xenopoulos, E., Mallouchos, A., Koutinas, A. A., Aggelis, G., et al. (2017). Lipid production and characterization by Mortierella (Umbelopsis) isabellina cultivated on lignocellulosic sugars. J. Appl. Microbiol. 123, 1461-1477. doi: 10.1111/jam.13587

Gidden, J., Denson, J., Liyanage, R., Ivey, D. M., and Lay, J. O. (2009). Lipid Compositions in Escherichia coli and Bacillus subtilis During Growth as Determined by MALDI-TOF and TOF/TOF Mass Spectrometry. Int. J. Mass. Spectrom. 283, 178-184. doi: 10.1016/j.ijms.2009.03.005

Grady, E. N., MacDonald, J., Liu, L., Richman, A., and Yuan, Z. -C. (2016). Current knowledge and perspectives of Paenibacillus: a review. Microb. Cell Fact. 15:203.
Gryganskyi, A. P., Golan, J., Dolatabadi, S., Mondo, S., Robb, S., Idnurm, A., et al. (2018). Phylogenetic and Phylogenomic Definition of Rhizopus Species. G3 118, 2007-2018.

Gurevich, A., Saveliev, V., Vyahhi, N., and Tesler, G. (2013). QUAST: quality assessment tool for genome assemblies. Bioinformatics 29, 1072-1075. doi: 10 . 1093/bioinformatics/btt086

Hanagasaki, T., and Asato, N. (2018). Changes in free amino acid content and hardness of beef while dry-aging with : changes in the quality of beef while dry-aging with. Hanguk Tongmul. Chawon. Kwahakhoe. Chi. 60:19.

Hermet, A., Méheust, D., Mounier, J., Barbier, G., and Jany, J. -L. (2012). Molecular systematics in the genus Mucor with special regards to species encountered in cheese. Fungal. Biol. 116, 692-705. doi: 10.1016/j.funbio.2012.04.002

Hildebrandt, U., Ouziad, F., Marner F. -J, and Bothe, H. (2006). The bacterium Paenibacillus validus stimulates growth of the arbuscular mycorrhizal fungus Glomus intraradices up to the formation of fertile spores. FEMS Microbiol. Lett. 254, 258-267.

Hoff, K. J., and Stanke, M. (2013). WebAUGUSTUS-a web service for training AUGUSTUS and predicting genes in eukaryotes. Nucleic Acids Res. 41, W123W128.

Huang, Q., An, H., Song, H., Mao, H., Shen, W., Dong, J. (2015). Diversity and biotransformative potential of endophytic fungi associated with the medicinal plant Kadsura angustifolia. Res. Microbiol. 166, 45-55. doi: 10.1016/j.resmic. 2014.12.004

Huang, Y. -S., Chaudhary, S., Thurmond, J. M., Bobik, E. G., Yuan, L., Chan, G. M., et al. (1999). Cloning of $\Delta 12$ - and $\Delta 6$-desaturases from Mortierella alpina and recombinant production of $\gamma$-linolenic acid in Saccharomyces cerevisiae. Lipids 410, 649-659. doi: 10.1007/s11745-999-0410-8

Ibrahim, A. S., and Spellberg, B. (2014). Zygomycetes as Agents of Infectious Disease in Humans. Mole. Princip. Fungal Pathog. 29, 428-440.

Ichihara, K., and Fukubayashi, Y. (2010). Preparation of fatty acid methyl esters for gas-liquid chromatography. J Lipid Res. 51, 635-640.

Idnurm, A., Walton, F. J., Floyd, A., and Heitman, J. (2008). Identification of the sex genes in an early diverged fungus. Nature 103, 193-196. doi: 10.1038/ nature06453

Ingham, C. J., Kalisman, O., Finkelshtein, A., and Ben-Jacob, E. (2011). Mutually facilitated dispersal between the nonmotile fungus Aspergillus fumigatus and the swarming bacterium Paenibacillus vortex. Proc. Natl. Acad. Sci. U. S. A. 108, 19731-19736. doi: 10.1073/pnas.1102097108

Itabangi, H., Sephton-Clark, P. C. S., Zhou, X., Insua, I., Probert, M., Correia, J., et al. (2019). A bacterial endosymbiont enables fungal immune evasion during fatal mucormycete infection. bioRxiv[Preprint] doi: 10.1101/584607

Janesch, B., Koerdt, A., Messner, P., and Schäffer, C. (2013). The S-Layer Homology Domain-Containing Protein SlhA from Paenibacillus alvei CCM 2051T Is Important for Swarming and Biofilm Formation. PLoS One. 66:e76566. doi: 10.1371/journal.pone.0076566

Janicki, T., Długoński, J., and Krupiński, M. (2018). Detoxification and simultaneous removal of phenolic xenobiotics and heavy metals with endocrine-disrupting activity by the non-ligninolytic fungus Umbelopsis isabellina. J. Hazard Mater. 360, 661-669. doi: 10.1016/j.jhazmat.2018.08.047

Jones, P., Binns, D., Chang H-Y, Fraser, M., Li, W., McAnulla, C., et al. (2014). InterProScan 5: genome-scale protein function classification. Bioinformatics 30, 1236-1240. doi: 10.1093/bioinformatics/btu031

Jorasch, P., Wolter, F. P., Zähringer, U., and Heinz, E. (1998). A UDP glucosyltransferase from Bacillus subtilis successively transfers up to four glucose residues to 1,2-diacylglycerol: expression of ypfP in Escherichia coli and structural analysis of its reaction products. Mol. Microbiol. 29, 419-430. doi: 10.1046/j.1365-2958.1998.00930.x

Kanehisa, M., Sato, Y., and Morishima, K. (2016). BlastKOALA and GhostKOALA: KEGG Tools for Functional Characterization of Genome and Metagenome Sequences. J. Mol. Biol. 428, 726-731. doi: 10.1016/j.jmb.2015.11.006

Katoh, K., and Standley, D. M. (2013). MAFFT Multiple Sequence Alignment Software Version 7: Improvements in Performance and Usability. Mol. Biol. Evol. 30, 772-780. doi: 10.1093/molbev/mst010

Kavermann, H., Burns, B. P., Angermuller, K., Odenbreit, S., Fischer, W., Melchers, K., et al. (2003). Identification and characterization of Helicobacter pylori genes essential for gastric colonization. J. Exp. Med. 197, 813-822. doi: 10.1084/jem. 20021531 
Kozlov, A. M., Darriba, D., Flouri, T., Morel, B., and Stamatakis, A. (2019). RAxML-NG: a fast, scalable and user-friendly tool for maximum likelihood phylogenetic inference. Bioinformatics 35, 4453-4455. doi: 10.1093/ bioinformatics/btz305

Laetsch, D. R., and Blaxter, M. L. (2017). BlobTools: Interrogation of genome assemblies. F1000Research. 2017:1287. doi: 10.12688/f1000research.12232.1

Lastovetsky, O. A., Gaspar, M. L., Mondo, S. J., LaButti, K. M., Sandor, L., Grigoriev, I. V., et al. (2016). Lipid metabolic changes in an early divergent fungus govern the establishment of a mutualistic symbiosis with endobacteria. Proc. Natl. Acad. Sci. U. S. A. 113, 15102-15107. doi: 10.1073/pnas.1615148113

Lebreton, A., Corre, E., Jany, J. -L., Brillet-Guéguen, L., Pèrez-Arques, C., Garre, V., et al. (2020). Comparative genomics applied to Mucor species with different lifestyles. BMC Genom. 21:135.

Lecointe, K., Cornu, M., Leroy, J., Coulon, P., and Sendid, B. (2019). Polysaccharides Cell Wall Architecture of Mucorales. Front. Microbiol. 2019:469. doi: 10.3389/fmicb.2019.00469

Lee, S. C., and Heitman, J. (2014). Sex in the Mucoralean fungi. Mycoses 57, 18-24. doi: $10.1111 /$ myc. 12244

Lee, S. C., and Idnurm, A. (2017). Fungal Sex: the Mucoromycota. Microbiol. Spectr. 5:1128. doi: 10.1128/microbiolspec.FUNK-0041-2017

Lefort, V., Desper, R., and Gascuel, O. (2015). FastME 2.0: A Comprehensive, Accurate, and Fast Distance-Based Phylogeny Inference Program: Table 1. Mole. Biol. Evol. 32, 2798-2800. doi: 10.1093/molbev/msv150

Linde, J., Schwartze, V., Binder, U., Lass-Flörl, C., Voigt, K., Horn, F. (2014). De Novo Whole-Genome Sequence and Genome Annotation of Lichtheimia ramosa. Genome Announc. 2:14. doi: 10.1128/genomeA.00888-14

Liu, G. -Q., and Jin, X. -C. (2008). Screening and optimization of microbial lipid production by Thamnidium sp., a novel oleaginous fungus isolated from forest soil. J. Biotechnol. 2008:S434. doi: 10.1016/j.jbiotec.2008.07.1005

Lu, S., Wang, J., Chitsaz, F., Derbyshire, M. K., Geer, R. C., Gonzales, N. R., et al. (2020). CDD/SPARCLE: the conserved domain database in 2020. Nucleic Acids Res. 48, D265-D268.

Lumini, E., Bianciotto, V., Jargeat, P., Novero, M., Salvioli, A., Faccio, A., et al. (2007). Presymbiotic growth and sporal morphology are affected in the arbuscular mycorrhizal fungus Gigaspora margarita cured of its endobacteria. Cell Microbiol. 9, 1716-1729. doi: 10.1111/j.1462-5822.2007.00907.x

Ma, L. -J, Ibrahim, A. S., Skory, C., Grabherr, M. G., Burger, G., Butler, M., et al. (2009). Genomic analysis of the basal lineage fungus Rhizopus oryzae reveals a whole-genome duplication. PLoS Genet. 5:e1000549. doi: 10.1371/journal.pgen. 1000549

Macdonald, R. M., and Chandler, M. R. (1981). Bacterium-Like Organelles In The Vesicular-Arbuscular Mycorrhizal Fungus Glomus Caledonius. N. Phytol. 1981, 241-246. doi: 10.1111/j.1469-8137.1981.tb07486.x

Maheshwari, R., Bharadwaj, G., and Bhat, M. K. (2000). Thermophilic Fungi: their Physiology and Enzymes. Microbiol. Mole. Biol. Rev. 64, 461-488. doi: 10.1128/ mmbr.64.3.461-488.2000

Maia, J. C. (1994). Hexosamine and cell wall biogenesis in the aquatic fungus Blastocladiella emersonii. FASEB J. 8, 848-853. doi: 10.1096/fasebj.8.11. 8070634

Markgraf, D. F., Klemm, R. W., Junker, M., Hannibal-Bach, H. K., Ejsing, C. S., Rapoport, T. A. (2014). An ER protein functionally couples neutral lipid metabolism on lipid droplets to membrane lipid synthesis in the ER. Cell. Rep. 6, 44-55. doi: 10.1016/j.celrep.2013.11.046

Meghwanshi, G. K., and Vashishtha, A. (2018). Biotechnology of Fungal Lipases. Fungi and their Role in Sustainable Development. Curr. Perspect. 2018, 383411. doi: 10.1007/978-981-13-0393-7_22

Meier-Kolthoff, J. P., and Göker, M. (2019). TYGS is an automated highthroughput platform for state-of-the-art genome-based taxonomy. Nat Commun. 10:2182.

Meier-Kolthoff, J. P., Auch, A. F., Klenk, H. -P, and Göker, M. (2013). Genome sequence-based species delimitation with confidence intervals and improved distance functions. BMC Bioinform. 14:60. doi: 10.1186/1471-2105-14-60

Mélida, H., Sain, D., Stajich, J. E., and Bulone, V. (2015). Deciphering the uniqueness of Mucoromycotina cell walls by combining biochemical and phylogenomic approaches. Environ. Microbiol. 17, 1649-1662. doi: 10.1111/ $1462-2920.12601$
Mondo, S. J., Lastovetsky, O. A., Gaspar, M. L., Schwardt, N. H., Barber, C. C., Riley, R., et al. (2017). Bacterial endosymbionts influence host sexuality and reveal reproductive genes of early divergent fungi. Nat. Commun. 8:1843.

Morin-Sardin, S., Nodet, P., Coton, E., and Jany, J. -L. (2017). Mucor: a Janus-faced fungal genus with human health impact and industrial applications. Fungal Biol. Rev. 31, 12-32. doi: 10.1016/j.fbr.2016.11.002

Mukherjee, K. D., and Kiewitt, I. (1987). Formation of .gamma.-linolenic acid in the higher plant evening primrose (Oenothera biennis L.). J. Agricult. Food Chem. 36, 1009-1012. doi: 10.1021/jf00078a036

Muszewska, A., Piłsyk, S., Perlińska-Lenart, U., and Kruszewska, J. S. (2017a). Diversity of Cell Wall Related Proteins in Human Pathogenic Fungi. J. Fungi. 4:6. doi: 10.3390/jof4010006

Muszewska, A., Steczkiewicz, K., and Ginalski, K. (2013). DIRS and Ngaro Retrotransposons in Fungi. PLoS One. 8:e76319. doi: 10.1371/journal.pone. 0076319

Muszewska, A., Steczkiewicz, K., Stepniewska-Dziubinska, M., and Ginalski, K. (2017b). Cut-and-Paste Transposons in Fungi with Diverse Lifestyles. Genome Biol. Evol. 9, 3463-3477. doi: 10.1093/gbe/evx261

Naranjo Ortiz, M. Á., and Others. (2019). Non-vertical genomics in fungal evolution. Fabra: Universitat Pompeu Fabra.

Navais, R., Méndez, J., Pérez-Pascual, D., Cascales, D., and Guijarro, J. A. (2014). The yrpAB operon of Yersinia ruckeri encoding two putative U32 peptidases is involved in virulence and induced under microaerobic conditions. Virulence 5, 619-624. doi: 10.4161/viru.29363

Neher, D. A., Weicht, T. R., Bates, S. T., Leff, J. W., and Fierer, N. (2013). Changes in bacterial and fungal communities across compost recipes, preparation methods, and composting times. PLoS One. 8:e79512. doi: 10.1371/journal.pone.0079512

Nemčovič, M., and Farkaš, V. (2001). Cell-Wall composition and polysaccharide synthhase activity changes following photoinduction in trichoderma viride. Acta Biol. Hungarica 2001, 281-288. doi: 10.1556/abiol.52.2001.2-3.12

Nichols, B. W., and Wood, B. J. B. (1968). The occurrence and biosynthesis of gamma-linolenic acid in a blue-green alga,Spirulina platensis. Lipids 68, 46-50. doi: 10.1007/bf02530968

Nicolás, F. E., Vila, A., Moxon, S., Cascales, M. D., Torres-Martínez, S., RuizVázquez, R. M., et al. (2015). The RNAi machinery controls distinct responses to environmental signals in the basal fungus Mucor circinelloides. BMC Genom. 16:237. doi: 10.1186/s12864-015-1443-2

Nordberg, H., Cantor, M., Dusheyko, S., Hua, S., Poliakov, A., Shabalov, I., et al. (2014). The genome portal of the Department of Energy Joint Genome Institute: 2014 updates. Nucleic Acids Res. 42, D26-D31.

Nout, M. J. R., Robert Nout, M. J., and Aidoo, K. E. (2010). Asian Fungal Fermented Food Industrial Applications. Netherland: Springer, 29-58.

Nykiel-Szymańska, J., Stolarek, P., and Bernat, P. (2018). Elimination and detoxification of 2,4-D by Umbelopsis isabellina with the involvement of cytochrome P450. Environ. Sci. Pollut. Res. Int. 25, 2738-2743. doi: 10.1007/ s11356-017-0571-4

Okrasińska, A., Bokus, A., Duk, K., Gęsiorska, A., Sokołowska, B., Miłobędzka, A., et al. (2021). New endosymbiotic relationships between Mucoromycota and Burkholderiaceae representatives. Appl. Environ. Microbiol. 22:AEM.02707-20. doi: 10.1128/AEM.02707-20

Orlowska, M., Steczkiewicz, K., and Muszewska, A. (2020). Utilization of cobalamin is ubiquitous in early-branching fungal phyla. bioRxiv[preprint]. doi: $10.1101 / 2020.10 .13 .337048$

Papanikolaou, S., and Aggelis, G. (2019). Sources of microbial oils with emphasis to Mortierella (Umbelopsis) isabellina fungus. World J. Microbiol. Biotechnol. 35:63.

Papanikolaou, S., Diamantopoulou, P., Chatzifragkou, A., Philippoussis, A., and Aggelis, G. (2010). Suitability of Low-Cost Sugars as Substrates for Lipid Production by the FungusThamnidium elegans. Energy Fuels. 2010, 4078-4086. doi: 10.1021/ef1004804

Partida-Martinez, L. P., and Hertweck, C. (2005). Pathogenic fungus harbours endosymbiotic bacteria for toxin production. Nature 437, 884-888. doi: 10 . 1038/nature03997

Partida-Martinez, L. P., Monajembashi, S., Greulich, K. -O., and Hertweck, C. (2007). Endosymbiont-dependent host reproduction maintains bacterial-fungal mutualism. Curr. Biol. 17, 773-777. doi: 10.1016/j.cub.2007.03.039 
Pawłowska, J., Okrasińska, A., Kisło, K., Aleksandrzak-Piekarczyk, T., Szatraj, K., Dolatabadi, S., et al. (2019). Carbon assimilation profiles of mucoralean fungi show their metabolic versatility. Sci. Rep. 9:11864.

Perlińska-Lenart, U., Piłsyk, S., Gryz, E., Turło, J., Hilszczańska, D., Kruszewska, J. S. (2020). Identification of bacteria and fungi inhabiting fruiting bodies of Burgundy truffle (Tuber aestivum Vittad.). Arch. Microbiol. 202, 2727-2738. doi: 10.1007/s00203-020-02002-x

Preston-Mafham, J., Boddy, L., and Randerson, P. F. (2002). Analysis of microbial community functional diversity using sole-carbon-source utilisation profiles - a critique. FEMS Microbiol. Ecol. 42, 1-14. doi: 10.1016/s0168-6496(02)00324-0

Qin, D., Wang, L., Han, M., Wang, J., Song, H., Yan, X., et al. (2018). Effects of an Endophytic Fungus Umbelopsis dimorpha on the Secondary Metabolites of Host-Plant Kadsura angustifolia. Front. Microbiol. 9:2845. doi: 10.3389/fmicb. 2018.02845

Rawlings, N. D., and Bateman, A. (2020). How to use the MEROPS database and website to help understand peptidase specificity. Protein Sci. 30, 83-92. doi: $10.1002 /$ pro.3948

Renne, M. F., and de Kroon, A. I. P. M. (2018). The role of phospholipid molecular species in determining the physical properties of yeast membranes. FEBS Lett. 592, 1330-1345. doi: 10.1002/1873-3468.12944

Richards, T. A., Leonard, G., and Wideman, J. G. (2017). What Defines the "Kingdom" Fungi? Microbiol. Spectr. 5:1128. doi: 10.1128/microbiolspec. FUNK-0044-2017

Richardson, M. (2009). The ecology of the Zygomycetes and its impact on environmental exposure. Clin. Microbiol. Infect. 72, 2-9. doi: 10.1111/j.14690691.2009.02972.x

Richardson, M. D., and Rautemaa-Richardson, R. (2019). Biotic Environments Supporting the Persistence of Clinically Relevant Mucormycetes. J. Fungi. 6. doi: 10.3390/jof6010004

Rivaldi, J. D., Carvalho, A. K. F., da Conceição, L. R. V., and de Castro, H. F. (2017). Assessing the potential of fatty acids produced by filamentous fungi as feedstock for biodiesel production. Preparat. Biochem. Biotechnol. 47, 970-976. doi: 10.1080/10826068.2017.1365246

Roukas, T., Varzakakou, M., and Kotzekidou, P. (2015). From cheese whey to carotenes by Blakeslea trispora in a bubble column reactor. Appl. Biochem. Biotechnol. 175, 182-193. doi: 10.1007/s12010-014-1260-0

Saier, M. H. Jr., Reddy, V. S., Tsu, B. V., Ahmed, M. S., Li, C., Moreno-Hagelsieb, G. (2016). The Transporter Classification Database (TCDB): recent advances. Nucleic Acids Res. 44, D372-D379.

Salvioli, A., Ghignone, S., Novero, M., Navazio, L., Venice, F., Bagnaresi, P., et al. (2016). Symbiosis with an endobacterium increases the fitness of a mycorrhizal fungus, raising its bioenergetic potential. ISME J. 10, 130-144. doi: 10.1038/ ismej.2015.91

Sasagawa, Y., Izaki, K., Matsubara, Y., Suzuki, K., Kojima, H., Kamio, Y. (1995). Molecular cloning and sequence analysis of the gene encoding the collagenase from Cytophaga sp. L43-1 strain. Biosci. Biotechnol. Biochem. 59, 2068-2073. doi: $10.1271 /$ bbb. 59.2068

Satari, B., and Karimi, K. (2018). Mucoralean fungi for sustainable production of bioethanol and biologically active molecules. Appl. Microbiol. Biotechnol. 102, 1097-1117. doi: 10.1007/s00253-017-8691-9

Schulz, E., Wetzel, J., Burmester, A., Ellenberger, S., Siegmund, L., and Wostemeyer, J. (2016). Sex loci of homothallic and heterothallic Mucorales. Endocytobiosis. Cell Res. 27, 39-57.

Schwartze, V. U., Winter, S., Shelest, E., Marcet-Houben, M., Horn, F., Wehner, S., et al. (2014). Gene expansion shapes genome architecture in the human pathogen Lichtheimia corymbifera: an evolutionary genomics analysis in the ancient terrestrial mucorales (Mucoromycotina). PLoS Genet. 10:e1004496. doi: 10.1371/journal.pgen.1004496

Seemann, T. (2014). Prokka: rapid prokaryotic genome annotation. Bioinformatics 30, 2068-2069. doi: 10.1093/bioinformatics/btu153

Seemann, T. (2019). abricate. GithubAbricate-Mass Screening of Contigs for Antimicrobial and Virulence Genes. Available online at: https://github.com/ tseemann/abricate [accessed on Aug 9, 2019].

Sharma, M., Schmid, M., Rothballer, M., Hause, G., Zuccaro, A., Imani, J., et al. (2008). Detection and identification of bacteria intimately associated with fungi of the orderSebacinales. Cell. Microbiol. 2008, 2235-2246. doi: 10.1111/j.14625822.2008.01202.x
Sheng, Y., Cong, J., Lu, H., Yang, L., Liu, Q., Li, D., et al. (2019). Broad-leaved forest types affect soil fungal community structure and soil organic carbon contents. Microbiologyopen 8:e874.

Simão, F. A., Waterhouse, R. M., Ioannidis, P., Kriventseva, E. V., and Zdobnov, E. M. (2015). BUSCO: assessing genome assembly and annotation completeness with single-copy orthologs. Bioinformatics 31, 3210-3212. doi: 10.1093/ bioinformatics/btv351

Simpson, J. T., Wong, K., Jackman, S. D., Schein, J. E., Jones, S. J. M., Birol, I. (2009). ABySS: a parallel assembler for short read sequence data. Genome Res. 108, 1117-1123. doi: 10.1101/gr.089532.108

Slater, G. S. C., and Birney, E. (2005). Automated generation of heuristics for biological sequence comparison. BMC Bioinform. 6:31.

Smit, A. F. A., Hubley, R., and Green, P. (2015). RepeatMasker Open-4.0. 20132015. URL: http://www.repeatmasker.org

Spatafora, J. W., Chang, Y., Benny, G. L., Lazarus, K., Smith, M. E., Berbee, M. L., et al. (2016). A phylum-level phylogenetic classification of zygomycete fungi based on genome-scale data. Mycologia 108, 1028-1046. doi: 10.3852/16-042

Stredansky, M., Conti, E., Stredanska, S., and Zanetti, F. (2000). $\gamma$-Linolenic acid production with Thamnidium elegans by solid-state fermentation on apple pomace. Bioresour. Technol. 2000, 41-45. doi: 10.1016/s0960-8524(99)00 132-7

Su, H., Blain, F., Musil, R. A., Zimmermann, J. J., Gu, K., and Bennett, D. C. (1996). Isolation and expression in Escherichia coli of hepB and hepC, genes coding for the glycosaminoglycan-degrading enzymes heparinase II and heparinase III, respectively, from Flavobacterium heparinum. Appl. Environ. Microbiol. 62, 2723-2734.

Takeda, I., Tamano, K., Yamane, N., Ishii, T., Miura, A., Umemura, M., et al. (2014). Genome Sequence of the Mucoromycotina Fungus Umbelopsis isabellina, an Effective Producer of Lipids. Genome Announc. 2:14. doi: 10.1128/genomeA. 00071-14

Tallant, C., García-Castellanos, R., Marrero, A., Canals, F., Yang, Y., Reymond, J. -L, et al. (2007). Activity of ulilysin, an archaeal PAPP-A-related gelatinase and IGFBP protease. Biol. Chem. 388, 1243-1253.

Tang, G. -L. (2018). Faculty Opinions recommendation of Resistance to nonribosomal peptide antibiotics mediated by D-stereospecific peptidases. Facul. Opin. Post Publ. Peer Rev. Biomed. Literat. 2018:905. doi: 10.3410/f. 732753547.793544905

Tang, X., Zhao, L., Chen, H., Chen, Y. Q., Chen, W., Song, Y., et al. (2015). Complete Genome Sequence of a High Lipid-Producing Strain of Mucor circinelloides WJ11 and Comparative Genome Analysis with a Low LipidProducing Strain CBS 277.49. PLoS One. 10:e0137543.

Tejesvi, M. V., Sauvola, T., Pirttilä, A. M., and Ruotsalainen, A. L. (2013). Neighboring Deschampsia flexuosa and Trientalis europaea harbor contrasting root fungal endophytic communities. Mycorrhiza 23, 1-10.

Uehling, J., Gryganskyi, A., Hameed, K., Tschaplinski, T., Misztal, P. K., Wu, S., et al. (2017). Comparative genomics of Mortierella elongata and its bacterial endosymbiont Mycoavidus cysteinexigens. Environ. Microbiol. 19, 2964-2983. doi: 10.1111/1462-2920.13669

Vamvakaki, A. -N., Kandarakis, I., Kaminarides, S., Komaitis, M., and Papanikolaou, S. (2010). Cheese whey as a renewable substrate for microbial lipid and biomass production by Zygomycetes. Engin. Life Sci. 2010, 348-360. doi: 10.1002/elsc.201000063

Větrovský, T., Morais, D., Kohout, P., Lepinay, C., Algora, C., Awokunle Hollá, S., et al. (2020). GlobalFungi, a global database of fungal occurrences from high-throughput-sequencing metabarcoding studies. Sci. Data 7:228.

Virgianti, D. P., and Natalia, D., (2020). Nyoman Pugeg Aryantha I. Short Communication: new record of Stenotrophomonas sp. as endosymbiont bacteria in Rhizopus microsporus. Biodiv. J. Biol. Div. 4:21. doi: 10.13057/ biodiv/d210449

Voigt, K., Wolf, T., Ochsenreiter, K., Nagy, G., Kaerger, K., Shelest, E., et al. (2016). "15 Genetic and Metabolic Aspects of Primary and Secondary Metabolism of the Zygomycetes," in Biochemistry and Molecular Biology. The Mycota (A Comprehensive Treatise on Fungi as Experimental Systems for Basic and Applied Research), ed, D. Hoffmeister (Cham: Springer).

Walther, G., Pawłowska, J., Alastruey-Izquierdo, A., Wrzosek, M., RodriguezTudela, J. L., Dolatabadi, S., et al. (2013). DNA barcoding in Mucorales: an inventory of biodiversity. Persoonia 30, 11-47. 
Wang, D., Li, M., Wei, D., Cai, Y. I., Zhang, Y., Xing, L. (2007). Identification and Functional Characterization of the Delta 6-Fatty Acid Desaturase Gene from Thamnidium elegans. J. Eukaryot. Microbiol. 136, 110-117. doi: 10.1111/j.15507408.2006.00136.x

Wetzel, J., Burmester, A., Kolbe, M., and Wöstemeyer, J. (2012). The matingrelated loci sexM and sexP of the zygomycetous fungus Mucor mucedo and their transcriptional regulation by trisporoid pheromones. Microbiology 158, 1016-1023.

Yang, S., Xu, W., Gao, Y., Chen, X., and Luo, Z. -H. (2020). Fungal diversity in deepsea sediments from Magellan seamounts environment of the western Pacific revealed by high-throughput Illumina sequencing. J. Microbiol. 58, 841-852.

Yin, Y., Mao, X., Yang, J., Chen, X., Mao, F., Xu, Y. (2012). dbCAN: a web resource for automated carbohydrate-active enzyme annotation. Nucleic Acids Res. 40, W445-W451.

Zdebska, E., and Kościelak, J. (1999). A single-sample method for determination of carbohydrate and protein contents glycoprotein bands separated by sodium dodecyl sulfate- polyacrylamide gel electrophoresis. Anal. Biochem. 275, 171179.

Zhang, H. -Z., and Chambers, H. F. (2004). BlaR1 and MecR1 gene products of Staphylococcus aureus. Handbook Proteol. Enzymes. 233, 769-771. doi: 10.1016/ b978-0-12-079611-3.50233-0
Zhu, Y., and Zhang, B. -B. (2013). Molecular cloning and functional characterization of a $\Delta 6$-fatty acid desaturase gene fromRhizopus oryzae. J. Basic Microbiol. 1002, 773-777. doi: 10.1002/jobm.201200189

Zikou, E., Chatzifragkou, A., Koutinas, A. A., and Papanikolaou, S. (2013). Evaluating glucose and xylose as cosubstrates for lipid accumulation and $\gamma$ linolenic acid biosynthesis of Thamnidium elegans. J. Appl. Microbiol. 114, 1020-1032.

Conflict of Interest: The authors declare that the research was conducted in the absence of any commercial or financial relationships that could be construed as a potential conflict of interest.

Copyright (C) 2021 Muszewska, Okrasińska, Steczkiewicz, Drgas, Orłowska, PerlińskaLenart, Aleksandrzak-Piekarczyk, Szatraj, Zielenkiewicz, Piłsyk, Malc, Mieczkowski, Kruszewska, Bernat and Pawłowska. This is an open-access article distributed under the terms of the Creative Commons Attribution License (CC BY). The use, distribution or reproduction in other forums is permitted, provided the original author(s) and the copyright owner(s) are credited and that the original publication in this journal is cited, in accordance with accepted academic practice. No use, distribution or reproduction is permitted which does not comply with these terms. 